DESY 11-094

KUNS-2340

June 15, 2011

\title{
Note on moduli stabilization, supersymmetry breaking and axiverse
}

\author{
Tetsutaro Higaki ${ }^{*}$ and Tatsuo Kobayashi2† \\ *DESY Theory Group, Notkestrasse 85, D-22607 Hamburg, Germany \\ ${ }^{\dagger}$ Department of Physics, Kyoto University, Kyoto 606-8502, Japan
}

\begin{abstract}
We study properties of moduli stabilization in the four dimensional $\mathcal{N}=1 \mathrm{su}$ pergravity theory with heavy moduli and would-be saxion-axion multiplets including light string-theoretic axions. We give general formulation for the scenario that heavy moduli and saxions are stabilized while axions remain light, assuming that moduli are stabilized near the supersymmetric solution. One can find stable vacuum, i.e. nontachyonic saxions, in the non-supersymmetric Minkowski vacua. We also discuss the cases, where the moduli are coupled to the supersymmetry breaking sector and/or moduli have contributions to supersymmetry breaking. Futhermore we study the models with axions originating from matter-like fields. Our analysis on moduli stabilization is applicable even if there are not light axion multiplets.
\end{abstract}

\footnotetext{
${ }^{1}$ E-mail address: tetsutaro.higaki@desy.de

${ }^{2}$ E-mail address: kobayash@gauge.scphys.kyoto-u.ac.jp
} 


\section{Introduction}

Moduli stabilization in superstring theories compactified on the internal space is necessary to determine physical parameters such as gauge couplings [1], Yukawa couplings [2, 3] and soft supersymmetry (SUSY) breaking parameters [4] in the visible sector, and to evade the moduli problem [5] and undesirable new forces [6]. As a consequence, it also can give several interesting implications to particle physics [7, 8, 9, 10, 11, 12, through the KKLT proposal 13] or the racetrack model [14].

The complex moduli fields in four dimension typically consist of scalars $\{\phi\}$ originating from geometry of compactification space (e.g. its volume) and pseudo-scalars $\{a\}$ coming from NSNS or RR tensor fields. Even though all the scalars $\{\phi\}$ are stabilized, some of their partners $\{a\}$ can still remain light due to the shift symmetries: $a \rightarrow a+$ const. Therefore the latter pseudo-scalars are often called string-theoretic axions [15, 16, 17] and can include the QCD axion to solve the strong CP problem [18, 19, 20] The number of these axions are originally determined by the topological property of compactified space, e.g. the Hodge numbers of CalabiYau (CY) three-fold [21]. (See also for effective field theories [22, 23].) Because the numbers can be much larger than of order unity, one can find many light string-theoretic axions through the moduli stabilization, that is, the string axiverse [24]. The axions can have large axion decay constants beyond the axion window 25] and can give influences on the cosmological observations [24]. For instance, their misalignment angles and Hubble scale during inflationary epoch are constrained and future observations of tensor modes and isocurvature perturbations could suggest the evidence of the (non-)axiverse [26]. Of course, the relic abundance of the axions should not exceed the observed matter density [27]. This will give interesting constraint not only on the observations but also on the string models in terms of moduli stabilization. Therefore our purpose is to study general framework of moduli stabilization leading to light axions based on the $\mathcal{N}=1$ supergravity (SUGRA).

Besides string-theoretic axions, one often obtains light field-theoretic axions at low energy, too. Thus, in general, the number of axions is estimated as 28]

$$
\begin{aligned}
\text { (the number of axions) }= & \text { (the number of fields }) \\
& +1-(\text { the number of terms in the } W) .
\end{aligned}
$$

\footnotetext{
${ }^{1}$ If we are to identify one of the axions with the QCD axion, the quality of the PQ symmetry needs to be checked for solving the strong CP problem: $\delta m_{a}^{2} \lesssim 10^{-11}\left(m_{a}^{\mathrm{QCD}}\right)^{2}$. Here axion mass $\delta m_{a}^{2}$ is a contribution from non-QCD effects, $m_{a}^{\mathrm{QCD}} \approx \Lambda_{\mathrm{QCD}}^{2} / f_{a}$ is the QCD axion mass just from the instanton, $f_{a}$ is the decay constant of the QCD axion and $\Lambda_{\mathrm{QCD}}=O(100) \mathrm{MeV}$ is the QCD scale.

${ }^{2}$ In the LARGE volume scenario [9], one can find $M_{\text {string }} \simeq 10^{11} \mathrm{GeV} \ll M_{\mathrm{Pl}}=2.4 \times 10^{18} \mathrm{GeV}$ [16].
} 
Here $W$ is the superpotential. This is because the Peccei-Quinn (PQ) shift symmetries of fields and the $R$-symmetry produce candidates of the axions whereas independent terms in the superpotential kill them, assuming the Kähler potential $K$ preserves these symmetries. Even if the $R$-symmetry is broken explicitly, this estimate is consistent when the constant in the superpotential is involved in the term "the number of terms in the $W$ ". Although we have neglected vector multiplets which can become massive, they can also reduce the number of axion candidates by absorbing them. When this counting becomes negative or zero, we do not have any light axions. If there are very small terms violating $\mathrm{PQ}$ symmetries in $W$ or $K$, they give very light masses to the axions.

In this paper, we study the moduli stabilization scenario leading to light axions. We discuss conditions to give heavy masses to all of real parts of moduli and leave some of imaginary parts massless. One of important conditions is SUSY breaking, and the typical mass scale is the gravitino mass $m_{3 / 2}$. All of the real parts of moduli must have masses, which are larger than the gravitino mass and/or comparable to the gravitino mass. On the other hand, light axions masses are smaller and could be of $\mathcal{O}\left(m_{3 / 2}^{r+1} / M_{p}^{r}\right)$ with $r=\mathcal{O}(1)$ or a few tens.

In Section 2, we will study the properties of non-supersymmetric vacua with light stringtheoretic axions. We will also give comments on closed string moduli which are directly coupled to the SUSY breaking sector. In Section 3, we will study the string-theoretic $R$-axion and the saxion-axion multiplet breaking SUSY. In Section 4, we will discuss corrections to the light axion masses from small breaking terms of PQ symmetries in the superpotential and the Kähler potential. In Section 5, we will give comments on simple models of field-theoretic axions in terms of effective field theories. In Section 6, we will conclude this paper. Our analysis on moduli stabilization is applicable even if there are not light axion multiplets. In Appendix, several types of moduli stabilization models are briefly reviewed. We will give a brief comment on the LARGE volume scenario based on the recent work of the neutral instanton effect including odd parity moduli under orientifold parity.

\section{Light string-theoretic axions}

In the following sections, we will consider moduli stabilization at low energy with the assumption that irrelevant moduli are heavy by closed string fluxes [29]. The remaining moduli of our interest can be stabilized via gaugino condensation [30] or (stringy) instanton effects [31]. Thus we study 
the superpotential below:

$$
W=W(\Phi)=W_{0}+\sum_{k} A_{k} \exp \left(-\sum_{i} a_{i}^{(k)} \Phi^{i}\right)
$$

Here $W_{0}$ is a constant from the fluxes, $\left\{\Phi^{i}\right\}$ are heavy closed string moduli fields which are stabilized by this superpotential and we use the unit $M_{\mathrm{Pl}}=2.4 \times 10^{18} \mathrm{GeV} \equiv 1$. We study the possibility that we can have massless axions at this stage. The scalar potential is written by the superpotential $W$ and the Kähler potential $K$,

$$
\begin{aligned}
V & =V_{F} \\
& =e^{G}\left[G_{I} G_{\bar{J}} G^{I \bar{J}}-3\right] \\
& =e^{K}\left[K^{I \bar{J}}\left(D_{I} W\right) \overline{\left(D_{J} W\right)}-3|W|^{2}\right],
\end{aligned}
$$

where

$$
G=K+\log |W|^{2}, \quad D_{I} W=\left(\partial_{I} K\right) W+\partial_{I} W .
$$

Here, $K^{I \bar{J}}=G^{I \bar{J}}$ denotes the inverse of the Kähler metric $K_{I \bar{J}}=\partial_{I} \bar{\partial}_{\bar{J}} K$. F-terms and the gravitino mass $m_{3 / 2}$ are given as

$$
F^{I}=-e^{G / 2} G^{I}=-e^{G / 2} G^{I \bar{J}} G_{\bar{J}}, \quad m_{3 / 2}=e^{G / 2} .
$$

We will focus just on $V_{F}$ for simplicity.

\subsection{Light string-theoretic axions and saxion masses in the SUSY vacuum}

In this subsection, we briefly review [16]. We study saxion masses in the SUSY vacuum with light axions.

For instance, let us consider the superpotential with two moduli $\left(T_{1}, T_{2}\right)$ :

$$
W=W_{0}+A e^{-a\left(T_{1}+T_{2}\right)} \equiv W_{0}+A e^{-a \Phi} .
$$

One can find $u \equiv T_{1}-T_{2}$ is absent from the superpotential, that is, we have just one phase of $\Phi$ : $\partial_{u} W=0$. Then the imaginary part $\operatorname{Im}(u)$ is a massless axion whereas $\operatorname{Re}(u)$ may be stabilized via the Kähler potential $K=K\left(T_{i}+\overline{T_{i}}\right)$.

One can generalize this argument to the case with many axions. Chiral superfields are classified into two classes. One class of fields $u^{\alpha}\left(\equiv \tau^{\alpha}+i b^{\alpha}\right)$ do not appear in the superpotential, i.e.

$$
\frac{\partial W}{\partial u^{\alpha}}=0
$$


while the fields $\Phi^{i}$ in the other class appear. Then, the imaginary parts of $u^{\alpha}$, i.e. $b^{\alpha}$ are string-theoretic axions, which have flat directions in the scalar potential for the form of Kähler potential, $K(u+\bar{u})$. We evaluate masses of the real parts of $u^{\alpha}$, i.e. saxions $\tau^{\alpha}$. In the SUSY vacuum with stabilized moduli one finds

$$
D_{\hat{i}} W=0 \quad \text { for }{ }^{\forall} \hat{i}=\left(\Phi^{i}, u^{\alpha}\right) .
$$

For the fields $u^{\alpha}$, this leads to

$$
\frac{\partial K}{\partial u^{\alpha}}=0 \quad \text { or } \quad W=0
$$

In this case, we find

$$
\left\langle\partial_{\tau^{\alpha}} \partial_{\tau^{\beta}} V_{F}\right\rangle_{\mathrm{SUSY}}=4 e^{K}|W|^{2}\left[2 K^{\hat{\hat{i}} \overline{\hat{j}}} K_{\hat{i}(\bar{\alpha}} K_{\beta) \overline{\hat{j}}}-3 K_{\alpha \bar{\beta}}\right]=-4 e^{K}|W|^{2} K_{\alpha \bar{\beta}} \leq 0 .
$$

That is, every massless string-theoretic axion has undesirable massless saxion for $W=0$ or tachyonic saxion in the SUSY AdS vacuum for $W \neq 0$. This is because $K_{\alpha \bar{\beta}}$ is the positive definite matrix. Note that the term $4 e^{K}|W|^{2} \cdot\left(-3 K_{\alpha \bar{\beta}}\right)$ comes from the vacuum energy. We have used the property of perturbative moduli Kähler potential,

$$
\partial_{\tau^{\alpha}} K(\Phi+\bar{\Phi} ; u+\bar{u})=2 \partial_{u^{\alpha}} K(\Phi+\bar{\Phi} ; u+\bar{u})=2 \partial_{\bar{u}^{\alpha}} K(\Phi+\bar{\Phi} ; u+\bar{u})
$$

The tachyonic instability might not be problematic in the AdS vacuum because of the Breitenlohner-Freedman bound [32]. At any rate, one should consider the SUSY breaking Minkowski vacuum to realize the realistic vacuum, although one may need fine-tuning to uplift the SUSY AdS vacuum to the Minkowski one. Hence, in the following sections, we will consider the SUSY breaking effects and then one can see that the saxions become stable for vanishing vacuum energy ${ }^{3}$.

\subsection{Light string-theoretic axions and the saxion mass in the SUSY breaking Minkowski vacuum}

Here, we study saxion stabilizaton in the SUSY breaking Minkowski vacuum with light axions. As a SUSY breaking source, we consider a single chiral field $X$. We assume that moduli $F$-terms $G^{\hat{i}}(\hat{i}=i, \alpha)$ are smaller than $G^{X}$ and the cosmological constant is vanishing, $\left\langle V_{F}\right\rangle=0$, that is,

$$
G^{X} G_{X} \simeq 3, \quad G^{X} G_{X} \gg G^{\hat{i}} G_{\hat{i}}
$$

\footnotetext{
${ }^{3}$ One can also consider a non-perturbative effect on the Kähler potential or D-term moduli stabilization which means a gauge multiplet eats an axion multiplet to lift saxion direction.
} 
where $G^{A}=G^{A \bar{B}} G_{\bar{B}}$.

Here, we study the model, where the SUSY breaking sector $X$ and moduli are decoupled in the Kähler potential $K$ and the superpotential $W$. That is, we consider the following form of the Kähler potential and the superpotential

$$
K=\hat{K}(X, \bar{X})+\mathcal{K}(\Phi+\bar{\Phi}, u+\bar{u}), \quad W=\hat{W}(X)+\mathcal{W}(\Phi)
$$

Hereafter we will set $K_{X \bar{X}}=1$ at the leading order of $\bar{X} X$. Note that $\partial_{\alpha} W=0$ and $G_{X \overline{\hat{i}}}=$ $K_{X \overline{\hat{i}}}=0$. When there is a large mass splitting between moduli $\Phi$ and $X, K_{X \bar{i}} \neq 0$ would be possible, but $K_{X \bar{i}} \ll 1$ would be necessary for the stable vacuum; $K_{X \bar{i}}=0$ would be an appropriate approximation. A simple example of the SUSY breaking models has $\hat{W}=\mu^{2} X$ [34, 35, 36, 37 3. At any rate, here we consider generic form of the SUSY breaking superpotential $\hat{W}$.

From the above assumption, one expects moduli $\Phi^{i}$ and $u^{\alpha}$ are stabilized near the SUSY solution,

$$
\mathcal{K}_{i} W+\mathcal{W}_{i} \sim 0, \quad \mathcal{K}_{\alpha} \sim 0
$$

such that one obtains heavier moduli masses than the gravitino mass $m_{3 / 2}=e^{G / 2}$. In the SUSY breaking vacuum with a vanishing cosmological constant, one finds the stationary condition:

$$
\partial_{I} V_{F}=G_{I} V_{F}+e^{G}\left[G_{I}+G^{K} \nabla_{I} G_{K}\right]=0
$$

which leads to

$$
G_{A I} G_{\bar{B}} G^{A \bar{B}}+G_{I}-G^{A} G^{\bar{B}} \partial_{I} G_{A \bar{B}}=0 .
$$

Here $I$ denotes $X, i, \alpha$ and $\nabla$ is a covariant derivative with respect to the Kähler metric. Since $G_{X \bar{i}}=0$, the above equation becomes

$$
\begin{aligned}
& \sqrt{3}\left(G_{X X}+1\right)+G_{X \hat{i}} G_{\bar{j}} G^{\hat{i} \overline{\hat{j}}}-G^{X} G^{\bar{X}} \partial_{X} G_{X \bar{X}}=0 \text { for } I=X, \\
& \sqrt{3} G_{X \hat{i}}+G_{\hat{i} \hat{j}} G_{\overline{\hat{k}}} G^{\hat{j} \bar{k}}+G_{\hat{i}}-G^{\hat{j}} G^{\overline{\hat{k}}} \partial_{\hat{i}} G_{\hat{j} \overline{\hat{k}}}=0 \text { for } I=\hat{i} .
\end{aligned}
$$

Here, we have used

$$
G_{X}=G_{\bar{X}}=\sqrt{3}
$$

\footnotetext{
${ }^{4}$ There are also models including SUSY breaking moduli [38], but we will not consider such models since subtle fine-tuning would be necessary.
} 
because $K_{X \bar{X}}=1$ and eq. (2.11). Using $G_{X \alpha}=0$, one finds in the vacuum

$$
G_{\alpha}=\mathcal{K}_{\alpha}=\frac{1}{2} G^{\hat{i}} G^{\overline{\hat{j}}} \partial_{\alpha} G_{\hat{i} \overline{\hat{j}}} .
$$

This means $F$-term of $u^{\alpha}$ is suppressed unless there is mixing between $\left(X, \Phi^{i}\right)$ and $u^{\alpha}$. For $X$ and $\Phi^{i}$, one can typically neglect sub-leading terms

$$
\begin{aligned}
& G_{X \hat{i}} G_{\bar{j}} G^{\hat{i} \overline{\hat{j}}} \ll 1, \\
& G_{i}-G^{\hat{j}} G^{\overline{\hat{k}}} \partial_{i} G_{\hat{j} \overline{\hat{k}}} \ll \sqrt{3} G_{X i}+G_{i \hat{j}} G_{\overline{\hat{k}}} G^{\hat{j} \overline{\hat{k}}},
\end{aligned}
$$

and one obtains

$$
\nabla_{X} G_{X} \simeq-1, \quad G^{i} \simeq-\sqrt{3}\left(G^{-1}\right)^{i j} G_{X j}
$$

Here $\left(G^{-1}\right)^{i j}(i, j \neq \alpha)$ is an inverse matrix of $G_{i j}=\mathcal{K}_{i \bar{j}}+\mathcal{W}_{i j} / W-\mathcal{W}_{i} \mathcal{W}_{j} / W^{2}$. Thus one can expect the shifts from the SUSY solution of $G^{i}=0$ and $\mathcal{K}_{\alpha}=0$ are given by

$$
\delta \Phi^{i} \sim \mathcal{K}_{\bar{k} l}\left(G^{-1}\right)^{i l}\left(\bar{G}^{-1}\right)^{\bar{k} \bar{j}} G_{\bar{X} \bar{j}}, \quad \delta u^{\alpha} \sim \mathcal{K}^{\alpha \bar{\beta}} G^{i} G^{\bar{j}} \bar{\partial}_{\bar{\beta}} G_{i \bar{j}}-\mathcal{K}^{\alpha \bar{\beta}} \mathcal{K}_{\bar{\beta} i} \delta \Phi^{i}
$$

Here we have used typical results $\sum_{\bar{k} \supset \text { heavy moduli }} \mathcal{K}_{i \bar{k}} \mathcal{K}^{\bar{k} j} \sim \delta_{i}^{j}$ and $\sum_{\bar{\gamma} \supset \text { light moduli }} \mathcal{K}_{\alpha \bar{\gamma}} \mathcal{K}^{\bar{\gamma} \beta} \sim \delta_{\alpha}^{\beta}$. One will see these shifts can be suppressed by the heavy moduli masses squared as $m_{3 / 2}^{2} / m_{\Phi^{i}}^{2}$.

\subsubsection{Masses for sGoldstino $X$ and heavy moduli $\Phi^{i}$}

We evaluate masses of $X$ and $\Phi^{i}$. By differentiating eq.(2.14), we obtain in the vacuum

$$
\begin{aligned}
\left\langle V_{I \bar{J}}\right\rangle & =e^{G}\left[G_{I \bar{J}}+\nabla_{I} G_{K} \bar{\nabla}_{\bar{J}} G^{K}-R_{I \bar{J} K \bar{L}} G^{K} G^{\bar{L}}\right]+\left(G_{I \bar{J}}-G_{I} G_{\bar{J}}\right) V_{F}, \\
\left\langle V_{I J}\right\rangle & =e^{G}\left[2 \nabla_{J} G_{I}+G^{K} \nabla_{J} \nabla_{I} G_{K}\right]+\left(\nabla_{J} G_{I}-G_{I} G_{J}\right) V_{F},
\end{aligned}
$$

where

$$
R_{I \bar{J} K \bar{L}} \equiv K_{I \bar{J} K \bar{L}}-K_{I K \bar{A}} K^{\bar{A} B} K_{\bar{J} \bar{L} B}
$$

Since we assumed that heavy moduli $\Phi^{i}$ are stabilized near the SUSY solution, one can neglect $G^{I}$ term to calculate heavy moduli masses $m_{\Phi^{i}}$ at the leading order of SUSY breaking effect.

For example, one expects

$$
m_{\Phi^{i}} \sim a_{i} \Phi^{i} m_{3 / 2}
$$

for the KKLT-like stabilization [13] and

$$
m_{\Phi^{i}} \gtrsim\left(a_{i} \Phi^{i}\right)^{2} m_{3 / 2},
$$


for the racetrack model [14, which is viable even for $W_{0}=0$. (See also Appendices A.2 and A.4 for the KKLT-like stabilization and the racetrack model, respectively.) Here $a_{i}$ denotes the most effective (or smallest) one in $\left\{a_{i}^{(k)}\right\}$ appearing in the eq. 2.1) to the moduli mass $m_{\Phi^{i}}$. One could obtain heavier moduli masses than the gravitino mass by fine-tuning the constant $W_{0}$ in the racetrack model [39].

In general, one expects $m_{\Phi^{i}} \gg m_{3 / 2}$ and mass squared matrix elements of the moduli $\Phi$ are written as

$$
V_{i \bar{j}} \simeq e^{G}\left[G_{i k} G_{\bar{j} \bar{l}} G^{k \bar{l}}\right] \equiv \mathcal{K}_{i \bar{j}} m_{\Phi^{i}}^{2}, \quad V_{i j} \sim 2 e^{G} G_{i j} \equiv 2 \mathcal{K}_{i \bar{j}} m_{3 / 2} m_{\Phi^{i}}
$$

that is,

$$
V_{i \bar{j}} \gg V_{i j},
$$

for $m_{\Phi^{i}} \gg m_{3 / 2}$. Note that the mass $\sqrt{V_{i \bar{j}} / \mathcal{K}_{i \bar{j}}} \simeq m_{\Phi^{i}}$ is the supersymmetric mass of modulus $\Phi^{i}$. In the above, we have used the following approximation,

$$
\begin{aligned}
G_{i j} & =\mathcal{K}_{i \bar{j}}+\frac{\mathcal{W}_{i j}}{W}-\frac{\mathcal{W}_{i} \mathcal{W}_{j}}{W^{2}} \simeq \mathcal{K}_{i \bar{j}}-\mathcal{K}_{i} \mathcal{K}_{j}+\frac{\mathcal{W}_{i j}}{W} \simeq \frac{\mathcal{W}_{i j}}{W} \equiv \mathcal{K}_{i j} \frac{m_{\Phi^{i}}}{m_{3 / 2}}, \\
G_{X i} & =-\frac{\mathcal{W}_{i} \hat{W}_{X}}{W^{2}} \simeq-\left(\sqrt{3}-\hat{K}_{X}\right) \mathcal{K}_{i} \sim \mathcal{K}_{i} \ll G_{i j}, \\
G_{i j k} & \sim \frac{\mathcal{W}_{i j k}}{W}-\frac{\mathcal{W}_{i j} \mathcal{W}_{k}}{W^{2}} \sim a_{k} \mathcal{K}_{i \bar{j}} \frac{m_{\Phi^{i}}}{m_{3 / 2}}+\mathcal{K}_{k} \mathcal{K}_{i \bar{j}} \frac{m_{\Phi^{i}}}{m_{3 / 2}} \sim a_{k} \mathcal{K}_{i \bar{j}} \frac{m_{\Phi^{i}}}{m_{3 / 2}} \\
G_{X i j} & \sim-\frac{W_{i j} \hat{W}_{X}}{W^{2}} \sim-\mathcal{K}_{i j} \frac{m_{\Phi^{i}}}{m_{3 / 2}} .
\end{aligned}
$$

We took the diagonal mass matrix $G_{i j}$ for simplifying the discussion here. Also one finds

$$
G^{i} \sim\left(3-\sqrt{3} \hat{K}_{X}\right) \mathcal{K}^{i \bar{j}} \mathcal{K}_{\bar{j}} \frac{m_{3 / 2}}{m_{\Phi^{i}}} \sim-\left(\Phi^{i}+\overline{\Phi^{i}}\right)\left(3-\sqrt{3} \hat{K}_{X}\right) \frac{m_{3 / 2}}{m_{\Phi^{i}}} .
$$

For $G^{\alpha}$ with $\mathcal{K}_{i \alpha} \neq 0$ for any $i$, their values are estimated as $G^{\alpha} \simeq \mathcal{K}^{\alpha \bar{i}} \bar{G}_{\bar{i}} \sim G^{i}$. Here we have used no-scale like structure $\sum_{\bar{j} \text { ’heavy moduli }} \mathcal{K}^{i \bar{j}} \mathcal{K}_{\bar{j}} \sim-\left(\Phi^{i}+\overline{\Phi^{i}}\right)$ up to would-be small perturbative corrections, though there is the small $u^{\alpha}$ dependencies $\mathcal{K}_{\alpha} \sim 0$. Note that the contribution of eq. 2.28) to $V_{i j}$ can be comparable to supersymmetric case, but one still has $V_{i \bar{j}} \gg V_{i j}$. Thus, one can obtain the (perturbatively) stable minimum for proper values of the moduli masses, $m_{\Phi^{i}}$. That is, by making $V_{i \bar{j}}$ larger than $V_{i j}$, one can realize positive definite mass eigenvalues for all of moduli around the SUSY solution $G^{i}=0$. Indeed, by using the above result, it is found the shift $\delta \Phi^{i}$ in 2.21) is suppressed by the factor, $m_{3 / 2}^{2} / m_{\Phi^{i}}^{2}$.

Next, we evaluate the mass of sGoldstino $X$. The sGoldstino acquires not the mass from $W$ but only SUSY breaking mass from the Kähler potential because of massless Goldstino in 
the rigid limit. There is the necessary condition (not sufficient) for the stable SUSY breaking vacuum, i.e. non-tachyonic non-holomorphic sGoldstino mass [38, 40]:

$$
m^{2}=V_{I \bar{J}} f^{I} f^{\bar{J}}=[3(1+\gamma) \hat{\sigma}-2 \gamma] m_{3 / 2}^{2}>0
$$

where

$$
\gamma \equiv \frac{V_{F}}{3 m_{3 / 2}^{2}}, \quad \hat{\sigma} \equiv \frac{2}{3}-R_{I \bar{J} K \bar{L}} f^{I} f^{\bar{J}} f^{K} f^{\bar{L}}, \quad f^{I} \equiv \frac{G^{I}}{\sqrt{G_{K} G^{K}}} .
$$

For $\gamma=0$ one expects

$$
\begin{aligned}
m^{2} & =3 \hat{\sigma} m_{3 / 2}^{2} \\
\hat{\sigma} & \simeq \frac{2}{3}-R_{X \bar{X} X \bar{X}}=\frac{2}{3}+K_{X X \bar{X}} K^{X \bar{X}} K_{\bar{X} \bar{X} X}-K_{X \bar{X} X \bar{X}} .
\end{aligned}
$$

For instance, let us consider the Kähler potential with a heavy scale $\Lambda \ll M_{\mathrm{Pl}} \equiv 1$ [41, 35, 36]

$$
\hat{K}=\bar{X} X-\frac{(\bar{X} X)^{2}}{4 \Lambda^{2}}+\cdots
$$

Then one obtains

$$
\hat{\sigma}=\frac{1}{\Lambda^{2}}+\frac{\bar{X} X}{\Lambda^{4}}+\frac{2}{3}>0 .
$$

Here $\langle X\rangle$ would be of $O\left(\Lambda^{2}\right)$ for the Polonyi model. For off-diagonal component $V_{X X}$, so long as $G_{X X X}$ and $\partial_{X} \Gamma_{X X}^{X}$ are of order unity in the Planck unit, one can find $V_{X X}=O\left(m_{3 / 2}^{2}\right) \ll m^{2}$. Thus, there would be the stable minimum. For string theories, $\Lambda$ would correspond to the mass scale of heavy field which is coupled to $X$, such as anomalous $U(1)$ gauge multiplet mass [42] which is comparable to the string scale, when $X$ has the $U(1)$ charge.

\subsubsection{Masses for saxion $\tau^{\alpha}$}

Here, we evaluate masses of saxion $\tau^{\alpha}$. One finds positive mass squared:

$$
\left\langle\partial_{\tau^{\alpha}} \partial_{\tau^{\beta}} V_{F}\right\rangle=4 e^{G}\left[2 G_{\alpha \bar{\beta}}-\partial_{\hat{i}} G_{\alpha \bar{\beta}} G^{\hat{i}}-\bar{\partial}_{\overline{\hat{i}}} G_{\alpha \bar{\beta}} G^{\overline{\hat{i}}}+G_{\hat{i}} G_{\overline{\hat{j}}} \partial_{\alpha} \bar{\partial}_{\bar{\beta}} G^{\hat{i} \overline{\hat{j}}}\right] \simeq 8 e^{G} G_{\alpha \bar{\beta}}>0
$$

Here we have neglected the last three terms in the bracket, since when one obtains $m_{3 / 2}=$ $e^{G / 2} \ll m_{\Phi^{i}}$ one can find

$$
G^{\hat{i}} \partial_{\hat{i}} G_{\alpha \bar{\beta}} \sim \frac{m_{3 / 2}}{m_{\Phi}} G_{\alpha \bar{\beta}}
$$

Again we have used no-scale like structure $\mathcal{K}_{\bar{j}} \mathcal{K}^{i \bar{j}} \mathcal{K}_{i}=$ const. Then the last three terms in eq.(2.35) are suppressed by $m_{3 / 2} / m_{\Phi}$ and $\left(m_{3 / 2} / m_{\Phi}\right)^{2}$ respectively, compared to the first term. 
Instead of $X$, with the sequestered explicit SUSY breaking term $V_{\text {lift }}=\epsilon e^{2 K / 3}$ where $\epsilon=$ $3\left\langle e^{K / 3}|W|^{2}\right\rangle$, one finds the similar results [17, $\left\langle\partial_{\tau^{\alpha}} \partial_{\tau^{\beta}} V_{F}\right\rangle \simeq 4 e^{G} G_{\alpha \bar{\beta}}$ and $G^{i} \sim\left(G^{-1}\right)^{i j} \mathcal{K}_{j} \sim$ $\left(\Phi^{i}+\overline{\Phi^{i}}\right) m_{3 / 2} / m_{\Phi^{i}}$, i.e. $m_{\alpha} \simeq \sqrt{2} m_{3 / 2}$. Here we have neglected the term which is proportional to $K_{\alpha} K_{\beta}$ in $\left\langle\partial_{\tau^{\alpha}} \partial_{\tau^{\beta}} V_{F}\right\rangle$. Note also that mass spectra of heavy moduli for such a case are similar to ones discussed above.

\subsubsection{Matrix elements}

Here, we summarize the mass matrix. Including other matrix elements, one can find typically

$$
\begin{aligned}
V_{i \bar{j}} & \sim e^{G}\left[G_{i k} G_{\bar{j}}^{k}+G_{i X} \bar{G}_{\bar{j} \bar{X}}\right] \sim e^{G} G_{i k} G_{\bar{j}}^{k} \simeq \mathcal{K}_{i \bar{j}} m_{\Phi^{i}}^{2}, \\
V_{i j} & \sim e^{G}\left[2 G_{i j}+G_{i j X}\right] \sim 2 e^{G} G_{i j} \simeq 2 \mathcal{K}_{i \bar{j}} m_{\Phi^{i}} m_{3 / 2}, \\
V_{i \bar{X}} & \sim e^{G}\left[G_{i j} G_{\bar{X}}^{j}+G_{i X}\right] \sim e^{G} G_{i j} G_{\bar{X}}^{j} \sim \mathcal{K}_{i} m_{\Phi^{i}} m_{3 / 2}, \\
V_{i X} & \sim e^{G} G^{j} G_{X i j} \sim m_{3 / 2}^{2} \frac{m_{\Phi^{i}}}{m_{\Phi^{j}}}\left(\Phi^{j}+\overline{\Phi^{j}}\right) \mathcal{K}_{i \bar{j}} \sim \mathcal{K}_{i} m_{3 / 2}^{2}, \\
V_{X \bar{X}} & \sim-e^{G} R_{X \bar{X} X \bar{X}}\left|G^{X}\right|^{2} \simeq 3 \hat{\sigma} m_{3 / 2}^{2}, \\
V_{X X} & \sim e^{G}\left[1+\nabla_{X} \nabla_{X} G_{X}+G^{i}\left(G_{X X i}+\Gamma_{X X}^{X} G_{X i}\right)\right] \sim m_{3 / 2}^{2}, \\
V_{i \tau^{\alpha}} & \sim e^{G} G_{k i} G^{j} G^{k \bar{m}} \mathcal{K}_{\alpha j \bar{m}} \sim m_{3 / 2}^{2} \frac{m_{\Phi^{i}}}{m_{\Phi^{j}}}\left(\Phi^{j}+\overline{\Phi^{j}}\right) \mathcal{K}_{i j \bar{\alpha}} \sim \mathcal{K}_{i \bar{\alpha}} m_{3 / 2}^{2}, \\
V_{X \tau^{\alpha}} & \sim e^{G} G_{i X} G^{j} G^{i \bar{m}} \mathcal{K}_{\alpha j \bar{m}} \sim\left(\Phi^{i}+\bar{\Phi}^{i}\right) \mathcal{K}_{i \bar{\alpha}} m_{3 / 2}^{2} \frac{m_{3 / 2}}{m_{\Phi^{i}}}, \\
V_{\tau^{\alpha} \tau^{\beta}} & \simeq 8 m_{3 / 2}^{2} \mathcal{K}_{\alpha \bar{\beta}},
\end{aligned}
$$

where we have used

$$
G_{X X i} \sim 2 \frac{W_{X}^{2} W_{i}}{W^{3}}-\frac{W_{X X} W_{i}}{W^{2}}-2 \frac{W_{X i} W_{X}}{W^{2}}+\frac{W_{X X i}}{W} \sim \mathcal{K}_{i}
$$

as well as $\partial_{X}^{n} W \lesssim W$. In general, $V_{X X}$ and $V_{i \bar{X}}$ could cause the vacuum instability even if $m_{\Phi^{i}} \gg m_{3 / 2}$ and $\hat{\sigma}>0$. Based on these matrix elements one expects the conditions

$$
\begin{aligned}
V_{i j} & <V_{i \bar{j}}, \quad V_{X X}<V_{X \bar{X}}, \\
V_{i X}, V_{i \bar{X}} & <\sqrt{V_{i \bar{j}} V_{X \bar{X}}}, \quad V_{i \tau^{\alpha}}<\sqrt{V_{i \bar{j}} V_{\alpha \bar{\beta}}}, \quad V_{X \tau^{\alpha}}<\sqrt{V_{X \bar{X}} V_{\tau^{\alpha} \tau^{\beta}}}
\end{aligned}
$$

should be satisfied for the (meta)stability. For this case, so long as $\hat{\sigma} \gg 1$ one would obtain the stable minimum. Then, the mass spectrum is summarized as

$$
m_{i}^{2} \simeq m_{\Phi^{i}}^{2} \gg m_{X^{ \pm}}^{2} \simeq 3 \hat{\sigma} m_{3 / 2}^{2}, \quad m_{\tau^{\alpha}}^{2} \simeq 4 m_{3 / 2}^{2}
$$

At this stage, the axions $b^{\alpha}$ are massless. Note that all of saxions $\tau^{\alpha}$ corresponding to massless axions have almost the same mass $m_{\tau^{\alpha}}=2 m_{3 / 2}$. 
Here, after the Goldstino is absorbed into the gravitino, the unnormalized axino masses are given by

$$
\begin{aligned}
\left(m_{\tilde{a}}\right)_{\alpha \beta} & =e^{G / 2}\left[\nabla_{\alpha} G_{\beta}+\frac{1}{3} G_{\alpha} G_{\beta}\right] \\
& \simeq e^{G / 2} G_{\alpha \bar{\beta}} .
\end{aligned}
$$

We have neglected $G_{\alpha}$ and $G_{i}$ because they are of $O\left(m_{3 / 2} / m_{\Phi^{i}}\right)$ corrections.

\subsubsection{F-term}

In the above case, one can find

$$
F^{X} \simeq-\sqrt{3} m_{3 / 2}, \quad \frac{F^{i}}{\Phi^{i}+\overline{\Phi^{i}}} \simeq \sqrt{3}\left(\sqrt{3}-K_{X}\right) m_{3 / 2} \frac{m_{3 / 2}}{m_{\Phi^{i}}} \sim \frac{F^{\alpha}}{u^{\alpha}+\overline{u^{\alpha}}} .
$$

Here we used the result

$$
G^{i} \simeq G^{i \bar{j}} G_{\bar{j}}, \quad G^{\alpha} \simeq G^{\alpha \bar{j}} G_{\bar{j}}, \quad(i, \bar{j} \neq \alpha),
$$

which leads to $G^{\alpha} \sim G^{i}$. Even if any $u^{\alpha}$ are stabilized via $D$-terms, $K_{\alpha} \sim 0$, we gain $F$-term of the $u^{\alpha}$ through the off-diagonal Kähler metric [43, 44, 45]. Note that if $G^{\alpha \bar{j}}=0$, one finds $F^{\alpha}=0$ since $G_{\alpha}=0$ for such a case 46]. For string-theoretic axion(s) breaking SUSY, see the Section 3.2.

\subsection{Note on mixing between $X$ and moduli and $D$-terms}

For simplicity, we have discussed so far the case that the SUSY breaking field $X$ does not couple to moduli $\Phi$ for a simplicity. However, in string theories, it is natural that moduli are coupled to the SUSY breaking sector via non-perturbative effects, so that one obtains much smaller scale than the string scale. Now, let us consider the mixing between $X$ and heavy moduli by replacing $\hat{W}(X)$ in (2.12) as follows,

$$
\hat{W}(X, \Phi)=f(X) \exp \left[-\sum_{i} a_{i}^{X} \Phi_{X}^{i}\right]
$$

Here $f(X)$ depends only on $X$. For instance one can consider the case that $f(X) \sim X$ [36, 37] or $f(X) \sim X^{-1}[10]$. Then, we consider the moduli stabilization with the superpotential,

$$
W=\hat{W}(X, \Phi)+\sum_{k} A_{k} e^{-\sum_{i} a_{i}^{(k)} \Phi_{X}^{i}}
$$

We assume

$$
a_{i}^{X} \sim a_{i},
$$


in the above superpotential, where $a_{i}$ is the most effective one to the moduli mass in $a_{i}^{(k)}$ for $\Phi_{X}^{i}$. Then one can find

$$
W_{X i} \sim-a_{i} W_{X} \simeq-a_{i}\left(\sqrt{3}-K_{X}\right) W
$$

Also, one obtains for $\Phi_{X}^{i}$

$$
\begin{aligned}
G_{X i} & =\frac{W_{X i}}{W}-\frac{W_{i} W_{X}}{W^{2}} \simeq-\left(\sqrt{3}-K_{X}\right)\left(a_{i}^{X}+\mathcal{K}_{i}\right) \lesssim G_{i j}, \\
G_{X i j} & \sim \frac{W_{X i j}}{W}-\frac{W_{i j} W_{X}}{W} \sim a_{i}^{X} a_{j}^{X}-\mathcal{K}_{i \bar{j}} \frac{m_{\Phi^{i}}}{m_{3 / 2}} \sim a_{i}^{X} a_{j}^{X} \gtrsim G_{i j}, \\
G_{X X i} & \sim 2 \frac{W_{X}^{2} W_{i}}{W^{3}}-\frac{W_{X X} W_{i}}{W^{2}}-2 \frac{W_{X i} W_{X}}{W^{2}}+\frac{W_{X X i}}{W} \sim \mathcal{K}_{i}+a_{i}^{X},
\end{aligned}
$$

and also we estimate

$$
\begin{aligned}
G^{i} & \sim\left(G^{-1}\right)^{i j} G_{j X} \sim \mathcal{K}^{i \bar{j}} a_{j}^{X} \frac{m_{3 / 2}}{m_{\Phi^{i}}} \sim a(\Phi+\bar{\Phi})^{2} \frac{m_{3 / 2}}{m_{\Phi}} \\
G^{\alpha} & \sim \mathcal{K}^{\alpha \bar{i}} \bar{G}_{\bar{i}} \sim G^{i}
\end{aligned}
$$

For metastability, one expects the conditions (2.39) should be satisfied.

Here, with the assumption that $G_{X X X}=O(1)$, one finds for $\Phi_{X}^{i}$

$$
\begin{aligned}
V_{i \bar{j}} & \sim e^{G}\left[G_{i k} G_{\bar{j}}^{k}+G_{i X} \bar{G}_{\bar{j} \bar{X}}\right] \sim \mathcal{K}_{i \bar{j}} m_{\Phi^{i}}^{2}+a_{i}^{X} a_{j}^{X} m_{3 / 2}^{2}, \\
V_{i j} & \sim e^{G}\left[2 G_{i j}+G_{i j X}+G^{k} G_{i j k}\right] \sim \mathcal{K}_{i \bar{j}} m_{\Phi^{i}} m_{3 / 2}+a_{i}^{X} a_{j}^{X} m_{3 / 2}^{2}+a_{i}^{X} a_{j} m_{3 / 2}^{2} \frac{m_{\Phi^{i}}}{m_{\Phi^{j}}} \\
V_{i \bar{X}} & \sim e^{G}\left[G_{i j} G_{\bar{X}}^{j}+G_{i X}\right] \sim a_{i}^{X} m_{\Phi^{i}} m_{3 / 2}+a_{i}^{X} m_{3 / 2}^{2}, \\
V_{i X} & \sim e^{G}\left[G_{X X i}+\left(1+\Gamma_{X X}^{X}\right) G_{X i}+G^{j} G_{X i j}\right] \sim a_{i}^{X} \mathcal{K}^{j \bar{k}} a_{j}^{X} a_{k}^{X} m_{3 / 2}^{2} \frac{m_{3 / 2}}{m_{\Phi^{j}}}+a_{i}^{X} m_{3 / 2}^{2}, \\
V_{X \bar{X}} & \sim G_{i X} G_{\bar{X}}^{i}-e^{G} R_{X \bar{X} X \bar{X}}\left|G^{X}\right|^{2} \simeq 3 \hat{\sigma}_{R} m_{3 / 2}^{2}+\mathcal{K}^{i \bar{j}} a_{i}^{X} a_{j}^{X} m_{3 / 2}^{2}, \\
V_{X X} & \sim e^{G}\left[1+\nabla_{X} \nabla_{X} G_{X}+G^{i}\left(G_{X X i}+\Gamma_{X X}^{X} G_{X i}\right)\right] \sim m_{3 / 2}^{2}\left(1+\mathcal{K}^{i \bar{j}} a_{i}^{X} a_{j}^{X} \frac{m_{3 / 2}}{m_{\Phi^{i}}}\right), \\
V_{i \tau^{\alpha}} & \sim e^{G}\left[G_{i \bar{\alpha}}+G_{k i} G^{j} G^{k \bar{m}} \mathcal{K}_{\alpha j \bar{m}}\right] \sim \mathcal{K}^{j \bar{k}} \mathcal{K}_{i j \bar{\alpha}} a_{k}^{X} m_{3 / 2}^{2} \frac{m_{\Phi^{i}}}{m_{\Phi^{j}}}+\mathcal{K}_{i \bar{\alpha}} m_{3 / 2}^{2} \\
& \sim \mathcal{K}^{j \bar{k}} \mathcal{K}_{i j \bar{\alpha}} a_{k}^{X} m_{3 / 2}^{2}+\mathcal{K}_{i \bar{\alpha}} m_{3 / 2}^{2}, \\
V_{X \tau^{\alpha}} & \sim e^{G} G_{i X} G^{j} G^{i \bar{m}} \mathcal{K}_{\alpha j \bar{m}} \sim \mathcal{K}^{i \bar{l}} a_{i}^{X} \mathcal{K}^{j \bar{m}} a_{m}^{X} \mathcal{K}_{j \bar{l} \alpha} m_{3 / 2}^{2} \frac{m_{3 / 2}}{m_{\Phi^{j}}} \\
V_{\tau^{\alpha} \tau^{\beta}} & \sim \mathcal{K}_{\alpha \bar{\beta}} m_{3 / 2}^{2}\left(8+a_{i}^{X} \Phi^{i} \frac{m_{3 / 2}}{m_{\Phi^{i}}}\right) \sim \mathcal{K}_{\alpha \bar{\beta}} m_{3 / 2}^{2},
\end{aligned}
$$

where $\hat{\sigma}_{R}$ denotes only $R_{X \bar{X} X \bar{X}}$ contribution in $\hat{\sigma}$.

However, if the linear combination of $a_{i}^{X} \Phi_{X}^{i}$ were stabilized via a KKLT-like model, i.e. $\left.D W\right|_{\mathrm{KKLT}} \sim 0$ and $m_{i} \sim\left(a_{i} \Phi_{X}^{i}\right) m_{3 / 2}$, one would obtain

$$
G^{X} G_{X} \sim G^{i} G_{i}
$$


in addition to $V_{i X} \sim V_{i \bar{X}} \sim \sqrt{V_{i \bar{j}} V_{X \bar{X}}}$ for $\hat{\sigma}_{R} \lesssim a_{i}^{X} \Phi_{X}^{i}, V_{i \bar{j}} \sim V_{i j}$ and $V_{i \tau^{\alpha}} \sim \sqrt{V_{i \bar{j}} V_{\tau^{\alpha} \tau^{\beta}}}$. This means the assumption that $G^{X}$ is the main source of the SUSY breaking is violated; KKLT stabilization of $a_{i}^{X} \Phi_{X}^{i}$ and realization of the Minkowski vacuum can not be realized successfully and the vacuum would be destabilized to the SUSY AdS one [36, 47, 48]. Even if the assumption that $a_{i} \sim a_{i}^{X}$ is violated, the uplifting to the Minkowski vacuum with KKLT stabilization of $a_{i}^{X} \Phi_{X}^{i}$ would fail since there would be the runaway direction, e.g., for small $X$. Thus, the linear combination of moduli $a_{i}^{X} \Phi_{X}^{i}$, which are coupled to the SUSY breaking sector $X$, should be stabilized via racetrack model [10, 36, 37], fluxes, or D-terms [49, 43], so that they gain much heavier masses than the KKLT-type mass, $m_{i} \gtrsim\left(a_{i} \Phi_{X}^{i}\right)^{2} m_{3 / 2} \gg\left(a_{i} \Phi_{X}^{i}\right) m_{3 / 2} \gg m_{3 / 2}$. (See also [28] for models in which there is the coupling between the SUSY breaking sector and the saxionaxion multiplet. In the model, one finds also the saxion mass much larger than the gravitino mass via the Kähler stabilization.)

For D-term stabilization $\partial_{\Phi^{i}} \mathcal{K}=0$, the moduli charged under anomalous $U(1)$ symmetries can become massive by U(1) symmetry breaking and the massive vector multiplet's eating them, even though $\partial_{\Phi^{i}} W=0$ if matter vevs become consequently irrelevant to the vector mass $M_{V}$ :

$$
m_{\Phi_{X}}^{2} \equiv M_{V}^{2} \simeq g^{2} \eta^{\Phi_{X}} \eta^{\Phi_{X}} \mathcal{K}_{\Phi_{X}} \overline{\Phi_{X}}
$$

Here $\eta^{\Phi_{X}}$ is the variation of $\Phi_{X}$ under the anomalous $U(1)$ and $M_{V}$ from $\Phi_{X}$ can be comparable to the string scale. Thus for such a case, one can find SUSY breaking Minkowski vacuum, i.e. via F-term [50] or D-term conditions [49, 43], the superpotential $W \sim A_{0}(\Psi) e^{-a_{i} \Phi^{i}}+e^{-a_{i}^{X} \Phi_{X}^{i}} X$ can be replaced by

$$
W \sim A_{0}(\langle\Psi\rangle) e^{-a_{i} \Phi^{i}}+e^{-a_{i}^{X}\left\langle\Phi_{X}^{i}\right\rangle} X \equiv A e^{-a_{i} \Phi^{i}}+\mu^{2} X
$$

in the low energy limit. Here $\{\Psi\}$ are open string modes. In the paper 43, when one obtains the tiny Fayet-Iliopoulos term

$$
\frac{M_{V}^{4}}{3} \simeq \xi_{\mathrm{FI}}
$$

so that $\Phi_{X}$ is absorbed into vector multiplet, one can find Minkowski vacuum due to the Polonyi model in the low energy limit. Here $\xi_{\mathrm{FI}}=\eta^{\Phi_{X}} \partial_{\Phi_{X}} \mathcal{K}$ is the Fayet-Iliopoulos term from moduli $\Phi_{X}$. For such a case, $F^{\Phi_{X}} \sim \eta^{\Phi_{X}} m_{3 / 2} \sim 10^{-2} m_{3 / 2}$ is obtained with D-term stabilization. (Note that one may find $M_{V}^{2} \ll \xi_{\mathrm{FI}}$ if $\partial_{\Phi_{X}}^{2} \mathcal{K} \sim \partial_{\Phi_{X}} \mathcal{K}$ and $\eta^{\Phi_{X}} \ll 1$.)

\footnotetext{
${ }^{5}$ For racetrack stabilization of $\Phi_{X}^{i}$, the condition that $V_{i \bar{X}}<\sqrt{V_{i \bar{j}} V_{X \bar{X}}}$ would be subtle for $\hat{\sigma}_{R} \lesssim a_{i}^{X} \Phi_{X}^{i}$. However, one can find the stable vacuum in the concrete models.
} 


\section{Approximate $R$-symmetry, $R$-axion and SUSY breaking mod- uli}

In this section, we study the model, which has an approximate $R$-symmetry and $R$-axion. We also study the model, where SUSY is also breaking by moduli fields. Indeed, we show that both models are investigated in the same way.

\section{1 $R$-axion and SUSY breaking moduli}

In general, a global $U(1) R$-symmetry is broken explicitly because string theory describes the quantum gravity. Indeed, string models with the exact and global $U(1) R$ symmetry have not been found. For instance the constant $W_{0}$ in the superpotential is easily obtained via flux compactifications, but the value depends on the choice of the flux vacua [51]. Therefore at a certain scale there may be an approximate $R$-symmetry accidentally in the SUSY breaking sector and the moduli stabilization sector when one obtains $W_{0}=0$ in the superpotential [52].

For example, the following superpotential,

$$
W=A e^{-a \Phi},
$$

has the R-symmetry, where the field $\Phi$ transforms as $\Phi \rightarrow \Phi-i \frac{2}{a} \alpha$ under the R-transformation with a transformation parameter $\alpha$. Similarly, the racetrack model has the R-symmetry [53, 36, 54, 10] if one has more than two fields in the superpotential without $W_{0}$. Thus, when $\operatorname{Re}(\Phi)$ is stabilized by the Kähler potential for example, we obtain the so-called light $R$-axion.

Here, we consider the R-symmetric superpotential. Then one can rewrite the superpotential including SUSY breaking sector $X$,

$$
W=e^{-\mathcal{R}} \mathcal{W}(X, \Phi),
$$

where $\partial_{\mathcal{R}} \mathcal{W}=0$. Since $\mathcal{R}$ can include not only $X$ but also moduli in the linear combination, we call it string-theoretic $R$-axion. Only $\mathcal{R}$ transforms as $\mathcal{R} \rightarrow \mathcal{R}+i 2 \alpha$ under the $R$-symmetry, while the others do not transform. Note that by the Kähler transformation with holomorphic function $\mathcal{G}$,

$$
K \rightarrow K+\mathcal{G}+\overline{\mathcal{G}}, \quad W \rightarrow \exp [-\mathcal{G}] W, \quad G \rightarrow G
$$

physics is invariant since the action is written by only the total Kähler potential $G=K+$

$\log |W|^{2}$. Thus one can consider the following Kähler potential $K$ and the superpotential $W$,

$$
K=K^{(0)}-(\mathcal{R}+\overline{\mathcal{R}}), \quad W=\mathcal{W}
$$


Here $K^{(0)}$ is the original Kähler potential obtained from the dimensional reduction. Then one finds

$$
G_{\mathcal{R}}=K_{\mathcal{R}}=K_{\mathcal{R}}^{(0)}-1, \quad G_{I}=K_{I}+\frac{\mathcal{W}_{I}}{\mathcal{W}} \quad \text { for } I \neq \mathcal{R}
$$

Hence unless $G_{\mathcal{R}}=0$, the $R$-axion is a source of the SUSY breaking. By Nelson and Seiberg argument [55, 54], the existence of the $R$-axion means the SUSY breaking, provided the model is generic and calculable. Hence we will also consider the SUSY breaking moduli with the vanishing cosmological constant: $G_{\mathcal{R}} \neq 0$ and $\left\langle V_{F}\right\rangle=0$.

Because the differences between string-theoretic $R$-axion and string-theoretic axions $u$ are just that the Kähler potential and their first derivatives as we saw, the following results are applicable not only to the string-theoretic $R$-axion, but also to usual string-theoretic axions $u$, which have non-trivial contributions to SUSY breaking.

\subsection{SUSY breaking string-theoretic $(R-)$ axions}

Let us consider the Kähler potential

$$
K=\hat{K}(X, \bar{X})+\tilde{K}(\mathcal{R}+\overline{\mathcal{R}})+\mathcal{K}(\Phi+\bar{\Phi})
$$

with $G_{\mathcal{R}} \neq 0$. For simplicity we will study the case that the Kähler potential is separable and focus only on the SUSY breaking string-theoretic $(R$-)axion neglecting dynamics of heavy moduli $\Phi$. Note that the discussion in this section is applicable to an usual string-theoretic axion $u$, which have non-trivial contributions to SUSY breaking.

One obtains the stationary condition with the vanishing cosmological constant:

$$
\begin{aligned}
\nabla_{X} G_{X} & \simeq-1 \\
G^{\mathcal{R}} & =\frac{G_{\mathcal{R}}}{\tilde{K}_{\mathcal{R} \overline{\mathcal{R}}}} \simeq 2 \frac{1}{\Gamma_{\mathcal{R} \mathcal{R}}^{\mathcal{R}}} \simeq 2 \frac{\tilde{K}_{\mathcal{R} \overline{\mathcal{R}}}}{\partial_{\mathcal{R}} \tilde{K}_{\mathcal{R} \overline{\mathcal{R}}}} \quad\left(\nabla_{\mathcal{R}} G_{\mathcal{R}}=-G_{\mathcal{R} \overline{\mathcal{R}}}\right)
\end{aligned}
$$

For the second derivatives $\partial_{I} \partial_{J} V_{F}$, we obtain

$$
\begin{aligned}
V_{X \bar{X}} & =e^{G}\left(2-R_{X \bar{X} X \bar{X}}\left|G^{X}\right|^{2}\right), \quad V_{r r}=4 e^{G}\left(2 \tilde{K}_{\mathcal{R} \overline{\mathcal{R}}}-R_{\mathcal{R} \overline{\mathcal{R}} \mathcal{R} \overline{\mathcal{R}}}\left|G^{\mathcal{R}}\right|^{2}\right), \\
V_{X X} & \sim e^{G}, \quad V_{r X}=0 .
\end{aligned}
$$

Here we have denoted $\mathcal{R}=r+i s$. When one sets $\hat{K}$ as (2.33), one obtains

$$
-R_{X \bar{X} X \bar{X}} \simeq \frac{1}{\Lambda^{2}} \gg 1 .
$$


With respect to the SUSY breaking $(R$-)axion, let us take the Kähler potential below

$$
\tilde{K} \equiv-n \log (\mathcal{R}+\overline{\mathcal{R}})+\delta(\mathcal{R}+\overline{\mathcal{R}})-(\mathcal{R}+\overline{\mathcal{R}})
$$

and we write

$$
\tilde{K}_{\mathcal{R} \overline{\mathcal{R}}} \equiv n \frac{[1+\Delta(\mathcal{R}+\overline{\mathcal{R}})]}{(\mathcal{R}+\overline{\mathcal{R}})^{2}}
$$

Then, one can find

$$
\begin{aligned}
G^{\mathcal{R}} & \simeq-(\mathcal{R}+\overline{\mathcal{R}})\left(1+\frac{1}{2}(\mathcal{R}+\overline{\mathcal{R}}) \cdot \Delta^{\prime}\right) \\
-R_{\mathcal{R} \overline{\mathcal{R}} \mathcal{R} \overline{\mathcal{R}}} & \simeq-2 n \frac{1}{(\mathcal{R}+\overline{\mathcal{R}})^{4}}\left(1+\Delta+\frac{1}{2}(\mathcal{R}+\overline{\mathcal{R}})^{2} \Delta^{\prime \prime}\right), \\
V_{r r} & \simeq-4 n \frac{\left(2 \Delta^{\prime}+(\mathcal{R}+\overline{\mathcal{R}}) \Delta^{\prime \prime}\right)}{(\mathcal{R}+\overline{\mathcal{R}})} e^{G} \sim \Delta \tilde{K}_{\mathcal{R} \overline{\mathcal{R}}} e^{G} .
\end{aligned}
$$

Here we used eq.(3.7) and $\Delta$ would come from the construction effect of $\mathcal{R}$ from the original moduli or the quantum effects of order $g_{s}$ and of order $\alpha^{\prime}$ and would be expected as

$$
\Delta \lesssim O(1)
$$

This result is applicable to many scenarios including the SUSY breaking light $(R$-)axion multiplet [9, 10, 53, 56]. For the above case, fine-tuning of the vanishing cosmological constant leads to

$$
\left|G_{X}\right|^{2}+G_{\mathcal{R}} G^{\mathcal{R}} \simeq\left|G_{X}\right|^{2}+n+O(\Delta)=3
$$

Then one should set

$$
G_{X} \simeq \sqrt{3-n+O(\Delta)}
$$

where $n>0$. Thus we obtain

$$
F^{X} \simeq-\sqrt{(3-n)+O(\Delta)} m_{3 / 2}, \quad \frac{F^{\mathcal{R}}}{\mathcal{R}+\overline{\mathcal{R}}} \simeq m_{3 / 2}, \quad \frac{F^{i}}{\Phi^{i}+\overline{\Phi^{i}}} \simeq m_{3 / 2} \frac{m_{3 / 2}}{m_{\Phi^{i}}} .
$$

For $n=3$, the sGoldstino is almost the SUSY breaking $(R$-)saxion.

Here non-holomorphic sGoldstino mass is given by

$$
\begin{aligned}
m^{2} & =3 \hat{\sigma} m_{3 / 2}^{2} \\
\hat{\sigma} & =\frac{2}{3}-\frac{1}{9}\left(R_{X \bar{X} X \bar{X}}\left|G^{X}\right|^{4}+R_{\mathcal{R} \overline{\mathcal{R}} \mathcal{R} \overline{\mathcal{R}}}\left|G^{\mathcal{R}}\right|^{4}\right) \\
& \sim \frac{2}{3}+\frac{1}{9 \Lambda^{2}}(3-n)^{2}-\frac{2 n}{9}+O(\Delta) .
\end{aligned}
$$


Then so long as $V_{r r}>0$, we would obtain positive definite mass matrix for $n \neq 3$

$$
m_{X^{ \pm}} \simeq \frac{m_{3 / 2}^{2}}{\Lambda^{2}}, \quad m_{r}^{2}=\frac{1}{2} \frac{V_{r r}}{\tilde{K}_{\mathcal{R} \overline{\mathcal{R}}}} \sim \Delta m_{3 / 2}^{2} .
$$

For $n=3$, one finds

$$
m_{X^{ \pm}} \sim m_{3 / 2}^{2}\left(1+\frac{\Delta}{\Lambda^{2}}\right), \quad m_{r}^{2} \sim \Delta m_{3 / 2}^{2}
$$

Here, after the Goldstino is absorbed into the gravitino, the unnormalized axino masses are given by

$$
\begin{aligned}
\left(m_{\tilde{a}}\right)_{\mathcal{R} \mathcal{R}} & =e^{G / 2}\left[\nabla_{\mathcal{R}} G_{\mathcal{R}}+\frac{1}{3} G_{\mathcal{R}} G_{\mathcal{R}}\right] \\
& \simeq e^{G / 2}\left[-G_{\mathcal{R} \overline{\mathcal{R}}}+\frac{1}{3} G_{\mathcal{R} \overline{\mathcal{R}}} G^{\mathcal{R}} G_{\mathcal{R}}\right] \\
& \simeq e^{G / 2} G_{\mathcal{R} \overline{\mathcal{R}}}\left[-1+\frac{n}{3}+O(\Delta)\right] .
\end{aligned}
$$

For $n=3$, SUSY breaking $(R$-)axino becomes the Goldstino, which is absorbed into the gravitino.

We give a comment on the small mixing $G_{\mathcal{R} i}=G_{\mathcal{R} \bar{i}} \neq 0$ here. In many cases, there is the off-diagonal Kähler metric $G_{\mathcal{R} i}=G_{\mathcal{R} \bar{i}} \neq 0$ and the main source of the SUSY breaking could be the overall (volume) modulus $(n=3)$ and it affects $F$-term of heavy moduli $\Phi^{i}$ if any: $G^{i} \sim\left(G^{-1}\right)^{i j} G^{\mathcal{R}} \nabla_{j} G_{\mathcal{R}} \sim\left(\Phi^{i}+\overline{\Phi^{i}}\right) m_{3 / 2} / m_{\Phi^{i}}$. Here we have used the explicit Kähler potential for the LARGE volume case in Appendix. However, as a consequence, the qualitative features in this section include such scenarios. Thus the result in this section would be applicable to such cases.

\section{Corrections to axion masses}

Axions $b^{\alpha}$ are exactly massless at the previous stage. Here, let us consider small corrections to the axion masses. These can be computed also in the SUSY vacuum, if the SUSY breaking sector does not violate any continuous PQ symmetry of $u^{\alpha}$. Recall that only heavy moduli should be coupled to the SUSY braking sector except the $R$-axion. For the small corrections, shifts of the saxion masses are negligible. 


\subsection{Superpotential correction}

Here, we consider the correction term $\delta W\left(\Phi^{i}, u\right)$ to the previous superpotential (2.1). That is, we study the following superpotential:

$$
W=\mathcal{W}\left(\Phi^{i}\right)+\delta W\left(\Phi^{i}, u\right)
$$

where

$$
\begin{aligned}
\mathcal{W}\left(\Phi^{i}\right) & =W_{0}+\sum_{k} A_{k} \exp \left(-\sum_{i} a_{i}^{(k)} \Phi^{i}\right), \\
\delta W\left(\Phi^{i}, u\right) & =\sum_{k} B_{k} \exp \left(-\sum_{\hat{i}} b_{\hat{i}}^{(k)} \Phi^{\hat{i}}\right) .
\end{aligned}
$$

Recall that $\Phi^{\hat{i}}$ denote all of the moduli including $\Phi^{i}$ and $u^{\alpha}$. Hence, the term $\mathcal{W}\left(\Phi^{i}\right)$ includes only heavy moduli $\Phi^{i}$, but not light axion multiplets $u^{\alpha}$, while $\delta W\left(\Phi^{i}, u\right)$ includes $u^{\alpha}$. We assume $B_{k} \simeq A_{k}=\mathcal{O}(1)$. We would like to consider the situation that $\langle\mathcal{W}\rangle \gg\langle\delta W\rangle$. If any terms $B_{k} \exp \left(-\sum_{\hat{i}} b_{\hat{i}}^{(k)} \Phi^{\hat{i}}\right)$ in $\delta W(\Phi, u)$ do not satisfy the condition, $\langle\mathcal{W}\rangle \gg B_{k} \exp \left(-\sum_{\hat{i}} b_{\hat{i}}^{(k)}\left\langle\Phi^{\hat{i}}\right\rangle\right)$, we have to take into account such terms from the previous stage of moduli stabilization in sections 2 and 3 and include them in $\mathcal{W}(\Phi)$. Then, some of $u$ become heavy reducing the number of light axions. Therefore heavy moduli should be coupled to saxion-axion multiplets in $\delta W$.

Then one finds the axion mass $m_{a}$ as 16, 26]

$$
\begin{aligned}
\mathcal{L} & =-K_{\alpha \bar{\beta}} \partial_{\mu} b^{\alpha} \partial^{\mu} b^{\beta}-\left(m_{a}^{2}\right)_{\alpha \beta} b^{\alpha} b^{\beta} \\
\left(m_{a}^{2}\right)_{\alpha \beta} & =3 e^{K}|W|^{2} \operatorname{Re}\left(\frac{\delta W_{\alpha \beta}}{W}\right),
\end{aligned}
$$

where $\delta W_{\alpha \beta} / W \gg \delta W_{\alpha} \delta W_{\beta} / W^{2}$ can be obtained in such vacua.

Now we parametrize $\delta W / W$, in particular, $b_{\hat{i}}^{(k)}\left\langle\Phi^{\hat{i}}\right\rangle \ln \langle\mathcal{W}\rangle$. For that purpose, we choose a typical term, say, $A_{j} \exp \left(-\sum_{i} a_{i}^{(j)} \Phi^{i}\right)$ in $\mathcal{W}$, which represents the value of $\langle\mathcal{W}\rangle$, i.e. $A_{j} \exp \left(-\sum_{i} a_{i}^{(j)}\left\langle\Phi^{i}\right\rangle\right) \sim$ $\mathcal{W}$. Then, we use the following parameters,

$$
r_{k}=\frac{\sum_{\hat{i}} b_{\hat{i}}^{(k)}\left\langle\Phi^{\hat{i}}\right\rangle}{\sum_{i} a_{i}^{(j)}\left\langle\Phi^{i}\right\rangle} .
$$

The parameters would satisfy $r_{k}>1$, because $\langle\mathcal{W}\rangle \gg\langle\delta W\rangle$. It is expected that $r_{k}$ is of $\mathcal{O}(1)$ or could be a few tens. Using these parameters, we write $B_{k} \exp \left(-\sum_{\hat{i}} b_{\hat{i}}^{(k)} \Phi^{\hat{i}}\right)$ in $\delta W$ as

$$
B_{k} \exp \left(-\sum_{\hat{i}} b_{\hat{i}}^{(k)} \Phi^{\hat{i}}\right) \simeq W\left(\frac{m_{3 / 2}}{M_{\mathrm{Pl}}}\right)^{r_{k}-1}
$$


Thus, the axion masses with the canonical normalization are given by

$$
\left(m_{a}^{2}\right)_{\alpha \alpha} \simeq 3 \frac{e^{K}|W|^{2}}{K_{\alpha \bar{\alpha}}} \operatorname{Re}\left(\frac{\delta W_{\alpha \alpha}}{W}\right) \simeq 3 \frac{b_{\alpha}^{2}}{f_{\alpha}^{2}} m_{3 / 2}^{2}\left(\frac{m_{3 / 2}}{M_{\mathrm{Pl}}}\right)^{r_{\alpha}-1},
$$

if and only if the axion mass is positive definite. Here we have defined through the diagonalization

$$
\delta W_{\alpha \alpha} \equiv b_{\alpha}^{2} \delta W, \quad K_{\alpha \bar{\alpha}} \equiv f_{\alpha}^{2}
$$

where $f_{\alpha}=O\left(M_{\text {string }} / M_{\mathrm{Pl}}\right)$ are diagonalized decay constants.

Once a small value of the gravitino mass $m_{3 / 2}$ is realized such as $m_{3 / 2} \ll M_{\mathrm{Pl}}$, the hierarchical axion masses with exponential suppression could appear. Some examples of mass scales are shown in Table 1 for $m_{3 / 2}=1,10$ and $100 \mathrm{TeV}$ up to $b_{\alpha}^{2} / f_{\alpha}^{2}$.

\begin{tabular}{|c|c|c|c|c|}
\hline$r_{\alpha}$ & 3 & 5 & 7 & 9 \\
\hline \hline$m_{a}$ for $m_{3 / 2}=1 \mathrm{TeV}$ & $10^{-4} \mathrm{eV}$ & $10^{-19} \mathrm{eV}$ & $10^{-34} \mathrm{eV}$ & $10^{-50} \mathrm{eV}$ \\
$m_{a}$ for $m_{3 / 2}=10 \mathrm{TeV}$ & $10^{-2} \mathrm{eV}$ & $10^{-16} \mathrm{eV}$ & $10^{-30} \mathrm{eV}$ & $10^{-45} \mathrm{eV}$ \\
$m_{a}$ for $m_{3 / 2}=100 \mathrm{TeV}$ & $1 \mathrm{eV}$ & $10^{-13} \mathrm{eV}$ & $10^{-26} \mathrm{eV}$ & $10^{-40} \mathrm{eV}$ \\
\hline
\end{tabular}

Table 1: Axion masses up to $b_{\alpha}^{2} / f_{\alpha}^{2}$.

We show several illustrating examples to lead to light axion masses in what follows.

- Example 0: $R$-axion mass

The small constant term in the superpotential induces the $R$-axion mass

$$
W=\mathcal{W} e^{-\mathcal{R}}+W_{0}
$$

For $W_{0} \ll \mathcal{W} e^{-\mathcal{R}} \sim A e^{-a \Phi}$, one finds

$$
\begin{aligned}
m_{a}^{2} & \sim e^{K} \frac{W_{0} \operatorname{Re}\left(e^{-\mathcal{R}} \mathcal{W}\right)}{\mathcal{K}_{\mathcal{R} \overline{\mathcal{R}}}}\left(\frac{K_{\mathcal{R}}}{\mathcal{K}_{\mathcal{R} \overline{\mathcal{R}}}}+O(1)\right) \\
& \sim \frac{m_{3 / 2}^{2}}{\mathcal{K}_{\mathcal{R} \overline{\mathcal{R}}}} \operatorname{Re}\left(\frac{W_{0}}{e^{-\mathcal{R} \mathcal{W}}}\right)\left(\frac{K_{\mathcal{R}}}{\mathcal{K}_{\mathcal{R} \overline{\mathcal{R}}}}+O(1)\right) .
\end{aligned}
$$

This result also coincides with the result of field-theoretic $R$-axion with $e^{-\mathcal{R}} \equiv \phi$ and $K=\bar{\phi} \phi$ even for larger $W_{0} \simeq \mathcal{W} e^{-\mathcal{R}}$. On the other hand, for $W_{0} \gtrsim \mathcal{W} e^{-\mathcal{R}}$, one finds the heavy $R$-axion like KKLT which is stabilized near the SUSY solution

$$
m_{a}^{2} \sim m_{3 / 2}^{2}\left(\frac{K_{\mathcal{R}}}{\mathcal{K}_{\mathcal{R} \overline{\mathcal{R}}}}\right)\left(\frac{K_{\mathcal{R}}}{\mathcal{K}_{\mathcal{R} \overline{\mathcal{R}}}}+O(1)\right) .
$$

Here one finds $\mathcal{W} e^{-\mathcal{R}} \sim \mathcal{K}_{\mathcal{R}} W_{0}$ for the KKLT stabilization.

\footnotetext{
${ }^{6}$ There will be also higer order terms from non-perturbative effects breaking the $R$-symmetry, such like $\omega e^{-2 \mathcal{R}}$ in the $\delta W$ where $\langle\omega\rangle \sim\left\langle\mathcal{W}^{2}\right\rangle$. But the discussion is similar to the case that $W_{0} \sim\left\langle\omega e^{-2 \mathcal{R}}\right\rangle$.
} 
- Example 1: $S U(N+M) \times S U(M)$ gaugino condensations or with an instanton $(M=1)$

Let us consider the KKLT type superpotential [16, 57]:

$$
W=W_{0}+e^{-a \Phi}+e^{-b(u+\Phi)}, \quad a=\frac{8 \pi^{2}}{N+M}, \quad b=\frac{8 \pi^{2}}{M}, \quad N \gg M,
$$

where $\Phi$ is the heavy modulus and $u$ is the light saxion-axion multiplet. In this case, assuming $\langle u\rangle \lesssim\langle\Phi\rangle$, one obtains $r \sim N / M+1$ and the axion mass is estimated as

$$
m_{a}^{2} \sim 3 \frac{b^{2}}{f^{2}} m_{3 / 2}^{2}\left(\frac{m_{3 / 2}}{M_{\mathrm{Pl}}}\right)^{\frac{N}{M}} .
$$

A similar result can be obtained in the racetrack model [26],

$$
\begin{aligned}
W & =W_{0}+e^{-a_{1} \Phi}-e^{-a_{2} \Phi}+e^{-b(u+\Phi)}, \\
a_{1,2} & =\frac{8 \pi^{2}}{N_{1,2}+M}, \quad b=\frac{8 \pi^{2}}{M}, \quad N_{1} \sim N_{2}, \quad N_{1,2} \gg M,
\end{aligned}
$$

when we do not fine-tune $W_{0}$ as a special value.

- Example 2: Many gaugino condensations or instantons wrapping on multiple cycles (in intersecting D-brane system)

Consider the superpotential with $n+3$ moduli; one is heavy modulus $\Phi$ and the remaining $n+2$ multiplets include light axions $u_{I}$

$$
\begin{aligned}
W= & W_{0}+e^{-a \Phi}+\sum_{i=1}^{n+2} \exp \left[-b_{i}\left(\sum_{I \neq i}^{n+2} u_{I}\right)-b \Phi\right], \\
& a \sim b_{i} \text { for }{ }^{\forall} i, \quad n \gg 1 .
\end{aligned}
$$

In this case, if $\left\langle u_{i}\right\rangle \sim\langle\Phi\rangle$ for ${ }^{\forall} i$, one finds $r \sim n+1$ and the axion mass is estimated as

$$
m_{a}^{2} \sim 3 \frac{b^{2}}{f^{2}} m_{3 / 2}^{2}\left(\frac{m_{3 / 2}}{M_{\mathrm{Pl}}}\right)^{n}
$$

However, if $\left\langle u_{i}\right\rangle \ll\langle\Phi\rangle$ for ${ }^{\forall} i$, one cannot obtain small axion masses; one needs $a \ll b$ as the previous example.

- Example 3: Including gaugino condensation on the magnetized brane

One may obtain the superpotential on the magnetized D7-branes or E3-branes wrapping on the divisor $D$ in type IIB orientifold:

$$
W=W_{0}+e^{-a \Phi}+\hat{B} e^{-b(u+\Phi)}, \quad \hat{B}=B \exp [-b \mathcal{M}\langle S\rangle]
$$


Here the constant $\mathcal{M}$ denotes $\mathcal{M}=\frac{1}{8 \pi^{2}} \int_{D} \mathcal{F}^{2} \in \mathbb{Z}$ up to curvature term [58], $\mathcal{F}$ is the world volume flux and $\langle S\rangle$ is the vev of the complex dilaton, which is fixed by three form flux. In this case, if $b\langle S\rangle \sim b\langle u\rangle \sim a\langle\Phi\rangle$, one can find $r \sim \mathcal{M}+1$ and the axion mass is estimated as

$$
m_{a}^{2} \sim 3 \frac{b^{2}}{f^{2}} m_{3 / 2}^{2}\left(\frac{m_{3 / 2}}{M_{\mathrm{Pl}}}\right)^{\mathcal{M}} .
$$

A value of $\mathcal{M}$ is weakly constrained via the tadpole condition of D3-branes in the F-theory limit of the orientifold compactification [59]:

$$
N_{D 3}+\frac{1}{2} N_{\text {flux }}(\mathcal{M})=\frac{\chi\left(Y_{4}\right)}{24} .
$$

Here $Y_{4}$ is an elliptically fibred Calabi-Yau four-fold. On the other hand, it would be natural and more plausible that $u \gtrsim \mathcal{M} S$ on the D7-brane, i.e. $\mathcal{M}=O(1)$. However, with the T-dual description, the present case would also be plausible since $\mathcal{M}$ corresponds to a winding number.

Thus, many models could lead to the hierarchical axion masses with suppression, $r=\mathcal{O}(1)$ or a few tens.

\subsection{Kähler potential correction}

Here, we comment on corrections to axion masses from the Kähler potential. Suppose that

$$
K=\mathcal{K}(\Phi+\bar{\Phi})+\delta K(\Phi, \bar{\Phi})
$$

where $\delta K(\Phi, \bar{\Phi})$ is a correction term and $\frac{\partial \delta K}{\partial b^{\alpha}} \neq 0$. Then one finds the axion masses 16

$$
\left(m_{a}^{2}\right)_{\alpha \beta}=3 e^{K}|W|^{2}\left[-\delta K_{\alpha \bar{\beta}}+\operatorname{Re}\left(\delta K_{\alpha \beta}\right)\right]
$$

Here we have neglected $O\left((\delta K)^{2}\right)$ term in the vacuum. It is plausible that $\delta K(\Phi, \bar{\Phi})$ would also appear from non-perturbative effects such as

$$
\delta K(\Phi, \bar{\Phi})=\sum_{k} B_{k}^{\prime} \exp \left(-\sum_{\hat{i}} b_{\hat{i}}^{\prime(k)} \Phi^{\hat{i}}\right)+\text { h.c. }
$$

In this case, the hierarchical axion masses with exponential suppression would be obtained similarly to the superpotential corrections. 


\section{Comment on axions from matter-like fields}

Here, we (briefly) study the superpotential including matter-like fields below:

$$
W=\mathcal{W}+\delta W
$$

where

$$
\begin{array}{r}
\mathcal{W} \equiv W_{0}+\sum_{k} A_{k}(\Psi) \exp \left(-\sum_{i} a_{i}^{(k)} \Phi^{i}\right) \\
\delta W \equiv \sum_{k} B_{k}(\Psi) \exp \left(-\sum_{i} b_{i}^{(k)} \Phi^{i}\right) .
\end{array}
$$

Here we omitted SUSY breaking sector and $\{\Psi\}$ means matter fields (or open string moduli) originating from the open string. We assume that the matter fields stabilized near the SUSY solution $K_{\Psi} W \sim W_{\Psi}$. Let us focus on the light matter-like fields whose axionic parts are massless while saxions are stabilized e.g. via $F$-term, $D$-term conditions or quantum radiative corrections. At low energy they are written by

$$
\begin{aligned}
\Psi^{P} & \equiv\left|\left\langle\Psi^{P}\right\rangle\right| e^{-\psi^{P}}, \\
A_{k}(\Psi) & =\prod_{P}\left|\left\langle\Psi^{P}\right\rangle\right| e^{-n_{P}^{k} \psi^{P}}, \quad B_{k}(\Psi)=\prod_{P}\left|\left\langle\Psi^{P}\right\rangle\right| e^{-m_{P}^{k} \psi^{P}} .
\end{aligned}
$$

Here some $A_{k}$ or $B_{k}$ can be constants. Consider linear combinations of $\hat{\psi}^{p}=\sum_{P} c_{P}^{p} \psi^{P}$, i.e. $\psi^{P}=\sum_{p}\left(c^{-1}\right)_{p}^{P} \hat{\psi}^{p} \rrbracket$, such that one can find

$$
\frac{\partial \mathcal{W}}{\partial \hat{\psi}^{p}}=0, \quad \frac{\partial A_{k}(\Psi)}{\partial \hat{\psi}^{p}}=0 \quad \text { for } \quad{ }^{\forall} k,{ }^{\exists} p .
$$

We use the Kähler potential at the tree level as

$$
K=\sum_{P} Z_{P}(\Phi+\bar{\Phi}) \overline{\Psi^{P}} \Psi^{P}
$$

Then one finds at low energy [28]

$$
\begin{aligned}
K & =\sum_{P} Z_{P}(\Phi+\bar{\Phi})\left|\left\langle\Psi^{P}\right\rangle\right|^{2} e^{-\left(\psi^{P}+\overline{\psi^{P}}\right)} \\
& \equiv \lambda_{p}\left(\hat{\psi}^{p}+\overline{\hat{\psi}^{p}}\right)+\frac{\lambda_{p q}}{2}\left(\hat{\psi}^{p}+\overline{\hat{\psi}^{p}}\right)\left(\hat{\psi}^{q}+\overline{\hat{\psi}^{q}}\right)+O\left((\hat{\psi}+\overline{\hat{\psi}})^{3}\right),
\end{aligned}
$$

where

$$
\lambda_{p}=-\sum_{P}\left(c^{-1}\right)_{p}^{P}\left|\left\langle\Psi^{P}\right\rangle\right|^{2}, \quad \lambda_{p q}=\sum_{P}\left(c^{-1}\right)_{p}^{P}\left(c^{-1}\right)_{q}^{P}\left|\left\langle\Psi^{P}\right\rangle\right|^{2} .
$$

\footnotetext{
${ }^{7}$ One can consider a linear combination including closed string moduli when matter-like fields are coupled to light moduli via a non-perturbative effect. For simplicity we will not consider such a case.
} 
For simplicity, we set $\lambda_{p}, \lambda_{p q}=$ const. in the vacuum, i.e. $Z_{P}=$ const. and would depend on much heavier moduli vevs. If $Z_{P}$ depends on the moduli or there are mixings between $\psi$ and $\Phi$ in $\hat{\psi}, \lambda_{p}$ and $\lambda_{p q}$ also have the dependence on the closed string moduli.

Then for axion multiplets one finds

$$
G_{p}=K_{p}=\lambda_{p}
$$

Thus even if $G_{p}=\lambda_{p}=0$ for axion multiplets $\hat{\psi}^{p}$, because of $\lambda_{p q} \neq 0$ one finds

$$
F^{p}=e^{G / 2} K^{p \bar{q}} G_{\bar{q}}=e^{G / 2}\left(\lambda^{-1}\right)^{p q} \lambda_{q} \sim m_{3 / 2},
$$

which leads to $F^{\Psi} / \Psi \sim m_{3 / 2}$. This result is consistent with the stationary condition $\partial_{p} V=0$ : $G_{p}+G^{q} \nabla_{p} G_{q}=0$, which leads to $G^{q}=O(1)$.

Then the saxion masses can be found

$$
\left\langle\partial_{\varphi^{p}} \partial_{\varphi^{q}} V_{F}\right\rangle=4 e^{G}\left[2 G_{p \bar{q}}-\partial_{r} G_{p \bar{q}} G^{r}-\bar{\partial}_{\bar{r}} G_{p \bar{q}} G^{\bar{r}}+G_{r} G_{\bar{s}} \partial_{p} \bar{\partial}_{\bar{q}} G^{r \bar{s}}\right] \sim e^{G} G_{p \bar{q}}
$$

where $\hat{\psi}^{p}=\varphi^{p}+i \vartheta^{p}$. Whether $\left\langle\partial_{\varphi^{p}} \partial_{\varphi^{q}} V_{F}\right\rangle>0$ or $<0$ depends on the model, but typical order of the saxion masses are of $O\left(m_{3 / 2}\right)$ even though the masses can also receive the contribution from D-terms of anomalous $U(1)$ symmetries 45 . When there is the vanishing saxion mass at the tree level, quantum radiative correction induces the mass smaller than $m_{3 / 2}$ [60, 61, 43]. Note that from the assumption that $Z_{P}=$ const., one finds $V_{\varphi^{p} X}=V_{\varphi^{p} i}=V_{\varphi^{p} \alpha}=0$.

The axion masses induced by $\delta W$ depend on the model, i.e. vevs of closed string moduli, those of matter like fields or the power of polynomial of matter-like fields in the superpotential. After the Goldstino is absorbed into the gravitino, the unnormalized axino masses are given by

$$
\begin{aligned}
\left(m_{\tilde{a}}\right)_{p q} & =e^{G / 2}\left[\nabla_{p} G_{q}+\frac{1}{3} G_{p} G_{q}\right] \\
& \sim e^{G / 2} G_{p \bar{q}} .
\end{aligned}
$$

\section{Conclusion and discussion}

We have studied properties of low energy moduli stabilization in the $\mathcal{N}=1$ effective SUGRA, which have heavy moduli and would-be saxion-axion multiplets. We have given general formulation for the scenario, where heavy moduli and saxions are stabilized and axions remain light.

\footnotetext{
8 For light non-axion multiplets which have of $O\left(m_{3 / 2}\right)$ masses, they can be stabilized through the superpotential $\left(\partial_{q} \mathcal{W} \neq 0\right)$ and have the similar properties to those of axion multiplets [43]. For instance one can obtain $G_{q}=K_{q}+\frac{W_{q}}{W} \sim \lambda_{q}$. Here $W_{q} \sim K_{q} W$. Therefore $\hat{\psi}^{p}$ s can include such light non-axion modes.
} 
SUSY breaking effects are important. In the non-supersymmetric Minkowski vacuum, the stable vacuum can be obtained even though there are light string-theoretic axions. In such a vacuum, heavy moduli and saxions can be stabilized supersymmetrically. In particular, saxions can be stabilized at the point $K_{\alpha} \sim 0$, while axions in the same multiplets remain lighter than the gravitino mass $m_{3 / 2}$. This scenario predicts the same number of saxions with the mass $2 m_{3 / 2}$ as the number of light axions. Note that our analysis on moduli stabilization is applicable even if there are not light axions in the vacuum.

When there are some moduli mixing the SUSY breaking source in the superpotential, such moduli would also destabilize the vacuum. In order to avoid such a situation, we need quite heavy masses for moduli. The moduli masses, which are generated in the KKLT-like model, are not enough, but one needs heavier masses, which would be generated through the racetrack model, D-term or closed string fluxes.

Alternatively, some moduli may contribute to SUSY breaking, e.g. the $R$-axion multiplet. In this case, the saxion mass can be lighter than the gravitino.

We have studied the effective SUGRA theory to lead to the axiverse. Following our realization, it is important to study further cosmological and particle phenomenological implications. In addition, our scenario predicts the same number of saxions with the mass $2 m_{3 / 2}$ as the number of light axions. These saxions would also have important implications depending on their masses, $2 m_{3 / 2}$. For example, when $m_{3 / 2}$ is around $\mathcal{O}(1)-\mathcal{O}(100) \mathrm{TeV}$, the late time entropy production by the vast number $(\sim 100)$ of saxion decays into radiations much before the BBN epoch can dilute harmful gravitino abundance [10] produced by decays of scalar fields such as heavy moduli [62]. (See [33, 63, 64] for discussions of the dilution by the SUSY breaking field $X$, which does not decay into gravitinos, based on the KKLT stabilization and see also [65, 66] for the relevant discussions.) It is interesting to study other aspects of axions and/or saxions following our realization of the axiverse.

We have discussed general aspects of low-energy effective SUGRA theory without fixing explicit string models. It is important to study explicit string model building leading to our scenario with moduli stabilization and light axions. We would study explicitly such string models elsewhere.

\section{Acknowledgement}

The authors would like to thank H.Kodama for useful discussions. The authors would like also to thank O. Lebedev for reading this maniscript and useful comments. T. K. is supported in part by the Grant-in-Aid for Scientific Research No. 20540266 and the Grant-in-Aid for the 
Global COE Program "The Next Generation of Physics, Spun from Universality and Emergence" from the Ministry of Education, Culture,Sports, Science and Technology of Japan. This work is supported by the JSPS Grant-in-Aid for Scientific Research (A) No. 22244030.

\section{A Moduli stabilization models}

Here we review several moduli stabilization models in type IIB Calabi-Yau O3/O7 orientifold models.

\section{A.1 D-term stabilization}

We show the relevant part of the model of the $D$-term stabilization. This is the model with the anomalous $U(1)$ gauge symmetry and e.g. the blowing-up mode 67]

$$
K=\frac{1}{2 \mathcal{V}_{E}}(M+\bar{M}+V)^{2}, \quad \partial_{M} W=0,
$$

where $V$ is the anomalous $U(1)$ vector multiplet and $\mathcal{V}_{E}$ is the compactification volume in the Einstein frame: $6 \mathcal{V}_{E}=\int J \wedge J \wedge J$, where $J$ is the Kähler form in the Einstein frame on the Calabi-Yau three-fold. One can ignore matter-like fields, depending on the charge signature of matter. Then one finds the minimum via SUSY condition $D_{M} W=D=K_{M}=(M+\bar{M}) / \mathcal{V}_{E}=0$ and obtains the massive vector multiplet $\tilde{V}=(M+\bar{M}+V)$, where $M$ is eaten by the gauge multiplet. The mass of the vector multiplet is now given by $g \mathcal{V}_{E}^{-1 / 2}$, where $g$ is the gauge coupling.

\section{A.2 KKLT}

We show the so-called KKLT model [13, 16] with

$$
K=-2 \log \left(\mathcal{V}_{E}\right), \quad W=W_{0}+\sum_{i}^{h_{+}^{1,1}} A_{i} e^{-a_{i} T^{i}}
$$

where $W_{0} \ll 1$. Here, $h_{+}^{1,1}\left(h_{-}^{1,1}\right)$ denotes the Hodge number of even (odd) parity moduli. To realize the SUSY breaking Minkowski vacuum, we add the uplifting potential,

$$
V_{\text {lift }}=\frac{\epsilon}{\mathcal{V}_{E}^{4 / 3}}, \quad \epsilon \simeq 3 \frac{\left|W_{0}\right|^{2}}{\left\langle\mathcal{V}_{E}^{2 / 3}\right\rangle}
$$


In this case, one finds

$$
\begin{aligned}
T_{i} & \simeq \frac{-1}{a_{i}} \log \left(W_{0}\right) \simeq \frac{1}{a_{i}} \log \left(\frac{M_{\mathrm{Pl}}}{m_{3 / 2}}\right), \\
\frac{F^{T_{i}}}{T_{i}+\overline{T_{i}}} & \simeq \frac{m_{3 / 2}}{a_{i} \tau_{i}} \simeq \frac{m_{3 / 2}}{\log \left(M_{\mathrm{Pl}} / m_{3 / 2}\right)},
\end{aligned}
$$

where $\tau_{i}=\operatorname{Re}\left(T_{i}\right)$. The gravitino mass and moduli masses are obtained as

$$
m_{3 / 2} \simeq \frac{W_{0}}{\mathcal{V}_{E}}, \quad m_{i} \simeq 2 a_{i} \tau_{i} m_{3 / 2}
$$

For one bulk volume modulus, we have

$$
\begin{aligned}
K & =-3 \log (T+\bar{T}), \quad W=W_{0}+e^{-a T}, \\
V_{\text {lift }} & =\frac{\epsilon}{(T+\bar{T})^{2}}, \quad \epsilon \simeq 3 \frac{\left|W_{0}\right|^{2}}{\langle(T+\bar{T})\rangle} .
\end{aligned}
$$

In this model, anomaly mediation is comparable to $F^{T} /(T+\bar{T})$. See also for generalization of this scenario 68.

\section{A.3 LARGE volume scenario}

This is the model [9] with bulk moduli and blowing-up modes, whose Kähler potential is written as

$$
K=-\log (S+\bar{S})-2 \log \left(\mathcal{V}_{E}+\frac{\hat{\xi}}{2}\right)
$$

with

$$
\mathcal{V}_{E}=\left(2 \tau_{b}\right)^{3 / 2}-\sum_{i}^{h_{+}^{1,1}-1}\left(2 \tau_{s, i}\right)^{3 / 2} .
$$

Here, for simplicity we have neglected moduli redefinitions at 1-loop level. The superpotential is written by

$$
W=W_{0}+\sum_{i}^{h_{+}^{1,1}-1} A_{i} e^{-a_{i} T_{s}^{i}},
$$

where $W_{0}=O(1)$, and the uplifting potential is added as,

$$
V_{\text {lift }}=\frac{\epsilon}{\mathcal{V}_{E}^{4 / 3}}, \quad \epsilon \simeq \frac{\left|W_{0}\right|^{2}}{8\left\langle\log \left(\mathcal{V}_{E}\right) \mathcal{V}_{E}^{5 / 3}\right\rangle}
$$


Note that $1 /\left(2 \pi g_{s}\right)=\operatorname{Re}(S)$. We can consider vanishing Standard Model (SM) cycle moduli or odd parity moduli with $D$-term stabilization: $K=\frac{1}{2 \mathcal{V}_{E}}(T+\bar{T})^{2}$. One can consider the $K 3$ fibration model: $\mathcal{V}_{E}=\tau_{b, 1} \tau_{b, 2}^{1 / 2}-\sum_{i}^{h_{+}^{1,1}-2} \tau_{s, i}^{3 / 2}$ together with loop corrections to fix bulk moduli.

Let us consider the simplest case, $h^{1,1}=h_{+}^{1,1}=2$. In this case, we have

$$
\begin{aligned}
\mathcal{V}_{E} & \simeq \tau_{b}^{3 / 2} \sim e^{a_{s} \tau_{s}}, \quad \tau_{s}^{3 / 2} \simeq \hat{\xi} \\
\frac{F^{T_{b}}}{T_{b}+\overline{T_{b}}} & \simeq m_{3 / 2}, \quad \frac{F^{T_{s}}}{T_{s}+\overline{T_{s}}} \simeq \frac{m_{3 / 2}}{\log \left(M_{\mathrm{Pl}} / m_{3 / 2}\right)} .
\end{aligned}
$$

Here, one finds

$$
m_{3 / 2} \simeq \frac{W_{0}}{\mathcal{V}_{E}}, \quad m_{b} \sim m_{3 / 2}\left(\frac{m_{3 / 2}}{M_{\mathrm{Pl}}}\right)^{1 / 2}, \quad m_{s} \sim \log \left(\mathcal{V}_{E}\right) m_{3 / 2},
$$

with $\mathcal{V}_{E} \gg 1$. Note $a_{s} \tau_{s} \sim \log \left(\mathcal{V}_{E}\right) \sim \log \left(M_{\mathrm{Pl}} / m_{3 / 2}\right)$ and $T_{b}$ is the SUSY breaking saxion similar to the case [56], whereas the axion is not couple to the visible sector. $T_{b}$ is an almost no-scale model modulus, while $T_{s}$ is a KKLT like modulus. In this model, anomaly mediation could be suppressed compared to $F^{T} /(T+\bar{T})$ by $\mathcal{V}_{E}^{-r}$, where $r$ is a fractional number.

\section{A.3.1 Modified original LARGE volume scenario}

Note that one can consider the model such like the original scenario with an additional odd parity moduli instead of vanishing the SM cycle on the D7-branes, i.e. $h_{+}^{1,1}=2$ and $h_{-}^{1,1}=1$ and we will discuss the neutral stringy instanton or gaugino condensation under the anomalous $U(1)$ symmetries on the brane with world volume flux [69]. This is the case in contrast to the paper [70] and is similar to the heterotic case [71]. Then, the Kähler potential and the superpotential are written by

$$
K=-\log (S+\bar{S})-2 \log \left(\mathcal{V}_{E}+\frac{\hat{\xi}}{2}\right), \quad W=W_{0}+A e^{-a\left(T_{+}+q G+h S\right)},
$$

where

$$
\mathcal{V}_{E}=\left(2 \tau_{b}\right)^{3 / 2}-\left(2 \tau_{+}+\frac{(G+\bar{G})^{2}}{(S+\bar{S})}\right)^{3 / 2} .
$$

Here $G$ is the odd parity Kähler moduli and note that in general odd parity moduli $\{G\}$ necessarily follow even parity moduli $\{T\}$ in the world volume of the brane. Then we took only the leading term of summation of instanton configuration for simplicity. In addition, the gauge kinetic function of the SM sector is written by

$$
f_{S M}=T_{+}+q_{S M} G+h_{S M} S .
$$


Again we neglect moduli redefinitions at 1-loop level. Here we assume that non-perturbative superpotential comes from the E3-brane instanton wrapping on the divisor $D_{E}$ with the flux. $h, q, h_{S M}$ and $q_{S M}$ depend on the flux on E3-brane and the visible sector D7-branes wrapping on $D_{S M}$ holding not only the SM gauge group but also the anomalous $U(1)$ symmetry respectively. $D_{E}$ and $D_{S M}$ map to $D_{E^{\prime}}$ and $D_{S M^{\prime}}$ respectively under orientifold action; $D_{E}$ and $D_{S M}$ include both even and odd elements, e.g. $\left[D_{E, S M}^{+}\right]=\left[D_{E, S M}\right]+\left[D_{E^{\prime}, S M^{\prime}}\right]$ and $\left[D_{E, S M}^{-}\right]=\left[D_{E, S M}\right]-$ $\left[D_{E^{\prime}, S M^{\prime}}\right]$, where $[D]$ is the Poincare dual of $D$. Here we take triple intersection $d_{b b b}=d_{+++}=$ $d_{+--}=1$ for simplicity. Now the presence of $G$ means there can be an anomalous $U(1)$ symmetry; both $T_{+}$and $G$ should be charged under the anomalous $U(1)$ symmetry:

$$
\delta G=i Q_{G}=i \frac{N}{8 \pi^{2}}, \quad \delta T_{+}=i Q_{T}=-i \frac{N}{8 \pi^{2}}\left(F_{D_{S M}}^{-}+\mathcal{F}_{D_{S M}}^{+}\right) .
$$

Here $N$ is the number of the D7-branes and $F=F_{D_{S M}}^{-} \omega_{-}+F_{D_{S M}}^{+} \omega_{+}$is the internal world volume flux relevant to the anomalous $U(1)$ on the visible sector D7-branes, where $\mathcal{F}^{+}=b^{+}+F^{+}$and $b^{+}=0$ or $1 / 2$ and $\omega_{-} \in H_{-}^{1,1}(C Y), \omega_{+} \in H_{+}^{1,1}(C Y)$ are (pull-back on the SM cycle of) the harmonic two-cycle basis on the CY space 5 . Here we took all the wrapping number of the D7brane and E3-brane against the even or odd cycle unity: $C_{E}^{+}=C_{E}^{-}=C_{D_{S M}}^{+}=C_{D_{S M}}^{-}=1$ in the notation of the paper [69]. Therefore, the following condition,

$$
q=F_{D_{S M}}^{-}+\mathcal{F}_{D_{S M}}^{+}
$$

should be satisfied for the neutral superpotential in this simple case $\square$. The $D$-term potential is given by

$$
V_{D}=\frac{1}{2 \operatorname{Re}(f)} D_{A}^{2}, \quad D_{A}=Q_{T} \partial_{T} K+Q_{G} \partial_{G} K=\frac{N}{8 \pi^{2}}\left(-q \partial_{T} K+\partial_{G} K\right),
$$

up to matter-like fields. If all the gauge couplings including the $U(1)$ symmetry are gauge invariant as the above simple case, the $U(1)$ can become non-anomalous; one should include matter. Otherwise, the $U(1)$ is in general anomalous; one would be able to neglect matter. For such a case, this model would have string theoretic axion, which is absorbed into the $U(1)$ vector multiplet. Define $\Phi \equiv T_{+}+q G$ and $u \equiv q T_{+}-G$. As a consequence $\Phi$ and $u$ are stabilized near SUSY solution without matter field vevs, $D_{\Phi} W \sim 0$ and $D_{A} \propto K_{u} \sim 0$; one obtains the scalar potential after integrating out $u$ and $\operatorname{Im}(\Phi)$ :

$$
V \simeq \frac{2 \sqrt{2} \sqrt{\phi} a^{2} \hat{A}^{2} e^{-2 a \operatorname{Re}(\Phi)}}{3 \mathcal{V}_{E}}-\frac{4 \phi a \hat{A} e^{-a \operatorname{Re}(\Phi)} W_{0}}{\mathcal{V}_{E}^{2}}+\frac{3 W_{0}^{2} \hat{\xi}}{2 \mathcal{V}_{E}^{3}},
$$

\footnotetext{
${ }^{9} b^{+}=1 / 2$ would be necessary because of the Freed-Witten anomaly 72 on the D7-branes wrapping on the $D_{S M}$ and the E3-brane.

${ }^{10}$ Here $f_{S M}$ could be also gauge invariant under the $U(1)$ since we could have $q=q_{S M}$; the $U(1)$ could be non-anomalous. However, for instance, when there is a relation that $C_{D_{S M}}^{-} \neq C_{E}^{+}$, or are fluxes depending on the SM gauge group and the $U(1), f_{S M}$ is not necessarily invariant under the $U(1): q \neq q_{S M}$ and the $U(1)$ is generally anomalous.
} 
where $\hat{A} \equiv A e^{-a h S}$. Here we have defined $\phi \equiv \operatorname{Re}(\Phi)-q^{2} / 8$. Thus one would find $m_{3 / 2} \sim \frac{W_{0}}{\mathcal{V}_{E}}$ and

$$
\begin{aligned}
\left\langle\mathcal{V}_{E}\right\rangle & \sim\left\langle\tau_{b}^{3 / 2}\right\rangle \sim e^{a(\Phi+h S)}, \quad\langle\Phi\rangle \sim \frac{\hat{\xi}^{2 / 3}}{2}+\frac{q^{2}}{8}+i \frac{\pi}{a}, \quad\langle\operatorname{Re}(u)\rangle \sim q\langle\operatorname{Re}(\Phi)\rangle-\frac{1}{4}\left(q^{3}+q\right), \\
m_{b} & \sim m_{3 / 2}\left(\frac{m_{3 / 2}}{M_{\mathrm{Pl}}}\right)^{1 / 2}, \quad m_{\Phi} \sim a \phi m_{3 / 2} \sim \log \left(\mathcal{V}_{E}\right) m_{3 / 2}, \quad m_{u}=M_{V} \sim Q \frac{M_{\mathrm{Pl}}}{\sqrt{\mathcal{V}_{E}}} \\
\frac{F^{b}}{T_{b}+\overline{T_{b}}} & \sim m_{3 / 2}, \quad \frac{F^{\Phi}}{2 \phi} \sim q^{-1} \frac{F^{u}}{2 \phi} \sim m_{3 / 2} \frac{m_{3 / 2}}{m_{\Phi}} \sim \frac{m_{3 / 2}}{\log \left(\mathcal{V}_{E}\right)}, \quad D_{A} \sim 0 .
\end{aligned}
$$

Thus we find $F^{T} \simeq F^{\Phi}$ and $F^{G} \simeq 0$. Here we have used $D_{A} \sim\left(\partial_{I} \bar{\partial}_{\bar{J}} D\right) F^{I} \bar{F}^{\bar{J}} / M_{V}^{2}$ [73, 74, 50, 43] and assumed that the anomalous $U(1)$ gauge coupling and vev of the $S$ are of $O(1)$. One finds in the vacuum $G^{u} \simeq q G^{\Phi}, \partial_{T_{b}} \bar{\partial}_{\bar{\Phi}} K_{u} \simeq-q \partial_{T_{b}} \bar{\partial}_{\bar{u}} K_{u}$ and $\partial_{\Phi} \bar{\partial}_{\bar{\Phi}} K_{u} \simeq-2 q \partial_{\Phi} \bar{\partial}_{\bar{u}} K_{u}$. Note also that $\partial_{T_{b}} \bar{\partial}_{\bar{T}_{b}} K_{u}$ and $\partial_{u} \bar{\partial}_{\bar{u}} K_{u}$ are irrelevant四 since one can obtain $K_{u} \sim 0$ in the vacuum; there is be a cancellation in the $D$-term at of $O\left(\mathcal{V}_{E}^{-2}\right)$ at least. Detailed study of this model is beyond the scope of this paper and we will leave it future work.

\section{A.4 Racetrack model}

This is the model [14] with bulk moduli and double gaugino condensations. The Kähler potential and the superpotential are obtained

$$
K=-2 \log \left(\mathcal{V}_{E}\right), \quad W=W_{0}+\sum_{i}^{h_{+}^{1,1}} A_{i} e^{-a_{i} T_{i}}-B_{i} e^{-b_{i} T_{i}},
$$

where $W_{0}<1$. Here, we add the uplifting potential,

$$
V_{\text {lift }}=\frac{\epsilon}{\mathcal{V}_{E}^{4 / 3}}, \quad \epsilon \simeq 3 \frac{\left\langle|W|^{2}\right\rangle}{\left\langle\mathcal{V}_{E}^{2 / 3}\right\rangle} .
$$

Then one finds via SUSY condition $D_{i} W \sim \partial_{i} W \sim 0$

$$
\begin{aligned}
T_{i} & \simeq \frac{1}{a_{i}-b_{i}} \log \left(\frac{a_{i} A_{i}}{b_{i} B_{i}}\right) \\
\frac{F^{T_{i}}}{T_{i}+\overline{T_{i}}} & \simeq \frac{m_{3 / 2}}{a_{i} b_{i} T_{i}^{2}} \simeq \frac{m_{3 / 2}^{2}}{m_{T_{i}}}, \quad m_{T_{i}} \simeq a_{i} b_{i}\left(T_{i}+\overline{T_{i}}\right)^{2} m_{3 / 2}^{2} .
\end{aligned}
$$

If one tunes $W_{0}$ to obtain $\langle W\rangle \sim 0$, moduli masses become much heavier than the gravitino mass 39.

\footnotetext{
11 Suppose that $D_{A} \sim K_{u} \lesssim \mathcal{V}_{E}^{-(1+1)} \ll \mathcal{V}_{E}^{-1}$. Then one can see $\partial_{T_{b}} \bar{\partial}_{\bar{T}_{b}} K_{u} \simeq K_{u} / \mathcal{V}_{E}^{4 / 3} \lesssim \mathcal{V}_{E}^{-(7 / 3+1)}$ and $\partial_{u} \bar{\partial}_{\bar{u}} K_{u} \sim K_{u} \lesssim \mathcal{V}_{E}^{-(1+1)}$ and they are negligible.
} 


\section{References}

[1] L. J. Dixon, V. Kaplunovsky and J. Louis, Nucl. Phys. B 355, 649 (1991); V. Kaplunovsky and J. Louis, Nucl. Phys. B 422, 57 (1994) [arXiv:hep-th/9402005]; V. Kaplunovsky and J. Louis, Nucl. Phys. B 444, 191 (1995) [arXiv:hep-th/9502077].

[2] S. Hamidi and C. Vafa, Nucl. Phys. B 279, 465 (1987); L. J. Dixon, D. Friedan, E. J. Martinec and S. H. Shenker, Nucl. Phys. B 282, 13 (1987). M. Cvetic and I. Papadimitriou, Phys. Rev. D 68, 046001 (2003) [Erratum-ibid. D 70, 029903 (2004)] [arXiv:hep-th/0303083]; S. A. Abel and A. W. Owen, Nucl. Phys. B 663, 197 (2003) [arXiv:hep-th/0303124]; S. A. Abel and A. W. Owen, Nucl. Phys. B 682, 183 (2004) [arXiv:hep-th/0310257]; D. Cremades, L. E. Ibanez and F. Marchesano, JHEP 0405, 079 (2004) [arXiv:hep-th/0404229]; K. S. Choi and T. Kobayashi, Nucl. Phys. B 797, 295 (2008) [arXiv:0711.4894 [hep-th]]; H. Abe, K. S. Choi, T. Kobayashi and H. Ohki, JHEP 0906, 080 (2009) [arXiv:0903.3800 [hep-th]].

[3] For recent discussions about Yukawa couplings in F-theory, for instance, see S. Cecotti, M. C. N. Cheng, J. J. Heckman and C. Vafa, arXiv:0910.0477 [hep-th]; J. P. Conlon and E. Palti, JHEP 1001, 029 (2010) [arXiv:0910.2413 [hep-th]]; H. Hayashi, T. Kawano, Y. Tsuchiya and T. Watari, JHEP 1008, 036 (2010) [arXiv:0910.2762 [hep-th]]; F. Marchesano and L. Martucci, Phys. Rev. Lett. 104, 231601 (2010) [arXiv:0910.5496 [hep-th]]; L. Aparicio, A. Font, L. E. Ibanez and F. Marchesano, arXiv:1104.2609 [hep-th].

[4] V. S. Kaplunovsky and J. Louis, Phys. Lett. B 306, 269 (1993) [arXiv:hep-th/9303040]; A. Brignole, L. E. Ibanez and C. Munoz, Nucl. Phys. B 422, 125 (1994) [Erratum-ibid. B 436, 747 (1995)] [arXiv:hep-ph/9308271].

[5] G. D. Coughlan, W. Fischler, E. W. Kolb, S. Raby and G. G. Ross, Phys. Lett. B 131, 59 (1983); T. Banks, D. B. Kaplan and A. E. Nelson, Phys. Rev. D 49, 779 (1994) [arXiv:hepph/9308292]; B. de Carlos, J. A. Casas, F. Quevedo and E. Roulet, Phys. Lett. B 318, 447 (1993) [arXiv:hep-ph/9308325].

[6] M. Cicoli, C. P. Burgess and F. Quevedo, arXiv:1105.2107 [hep-th]; references therein.

[7] K. Choi, A. Falkowski, H. P. Nilles and M. Olechowski, Nucl. Phys. B 718, 113 (2005) [arXiv:hep-th/0503216]; K. Choi, K. S. Jeong and K. i. Okumura, JHEP 0509, 039 (2005) [arXiv:hep-ph/0504037]; M. Endo, M. Yamaguchi and K. Yoshioka, Phys. Rev. D 72, 015004 (2005) [arXiv:hep-ph/0504036]. 
[8] A. Falkowski, O. Lebedev and Y. Mambrini, JHEP 0511, 034 (2005) [arXiv:hepph/0507110]; K. Choi, K. S. Jeong, T. Kobayashi and K. i. Okumura, Phys. Lett. B 633, 355 (2006) [arXiv:hep-ph/0508029]; R. Kitano and Y. Nomura, Phys. Lett. B 631, 58 (2005) [arXiv:hep-ph/0509039]; H. Baer, E. K. Park, X. Tata and T. T. Wang, JHEP 0608, 041 (2006) [arXiv:hep-ph/0604253]; K. Choi, K. S. Jeong, T. Kobayashi and K. i. Okumura, Phys. Rev. D 75, 095012 (2007) [arXiv:hep-ph/0612258]; H. Abe, Y. G. Kim, T. Kobayashi and Y. Shimizu, JHEP 0709, 107 (2007) [arXiv:0706.4349 [hep-ph]].

[9] V. Balasubramanian, P. Berglund, J. P. Conlon and F. Quevedo, JHEP 0503, 007 (2005) [arXiv:hep-th/0502058]; J. P. Conlon, F. Quevedo and K. Suruliz, JHEP 0508, 007 (2005) [arXiv:hep-th/0505076]; J. P. Conlon and F. Quevedo, JHEP 0606, 029 (2006) [arXiv:hepth/0605141]; J. P. Conlon, S. S. Abdussalam, F. Quevedo and K. Suruliz, JHEP 0701, 032 (2007) [arXiv:hep-th/0610129]; M. Berg, M. Haack and E. Pajer, JHEP 0709, 031 (2007) [arXiv:0704.0737 [hep-th]]; J. P. Conlon and F. Quevedo, JCAP 0708, 019 (2007) [arXiv:0705.3460 [hep-ph]]; M. Cicoli, J. P. Conlon and F. Quevedo, JHEP 0801, 052 (2008) [arXiv:0708.1873 [hep-th]]. M. Cicoli, J. P. Conlon and F. Quevedo, JHEP 0810, 105 (2008) [arXiv:0805.1029 [hep-th]]; R. Blumenhagen, J. P. Conlon, S. Krippendorf, S. Moster and F. Quevedo, JHEP 0909, 007 (2009) [arXiv:0906.3297 [hep-th]]; J. P. Conlon and F. G. Pedro, JHEP 1006, 082 (2010) [arXiv:1003.0388 [hep-th]]. K. Choi, H. P. Nilles, C. S. Shin and M. Trapletti, JHEP 1102, 047 (2011) [arXiv:1011.0999 [hep-th]].

[10] B. S. Acharya, K. Bobkov, G. Kane, P. Kumar and D. Vaman, Phys. Rev. Lett. 97, 191601 (2006) [arXiv:hep-th/0606262]; B. S. Acharya, K. Bobkov, G. L. Kane, P. Kumar and J. Shao, Phys. Rev. D 76, 126010 (2007) [arXiv:hep-th/0701034]; B. S. Acharya, K. Bobkov, G. L. Kane, J. Shao and P. Kumar, Phys. Rev. D 78, 065038 (2008) [arXiv:0801.0478 [hepph]]; B. S. Acharya, P. Kumar, K. Bobkov, G. Kane, J. Shao and S. Watson, JHEP 0806, 064 (2008) [arXiv:0804.0863 [hep-ph]].

[11] S. A. Abel, J. Jaeckel, V. V. Khoze and A. Ringwald, Phys. Lett. B 666, 66 (2008) [arXiv:hep-ph/0608248]; S. A. Abel, M. D. Goodsell, J. Jaeckel, V. V. Khoze and A. Ringwald, JHEP 0807, 124 (2008) [arXiv:0803.1449 [hep-ph]]; M. Goodsell, J. Jaeckel, J. Redondo and A. Ringwald, JHEP 0911, 027 (2009) [arXiv:0909.0515 [hep-ph]]; M. Cicoli, M. Goodsell, J. Jaeckel and A. Ringwald, arXiv:1103.3705 [hep-th]; M. Williams, C. P. Burgess, A. Maharana and F. Quevedo, arXiv:1103.4556 [hep-ph].

[12] J. J. Blanco-Pillado et al., JHEP 0411, 063 (2004) [arXiv:hep-th/0406230]; J. J. BlancoPillado et al., JHEP 0609, 002 (2006) [arXiv:hep-th/0603129]; J. P. Conlon and F. Quevedo, 
JHEP 0601, 146 (2006) [arXiv:hep-th/0509012]; M. Cicoli, C. P. Burgess and F. Quevedo, JCAP 0903, 013 (2009) [arXiv:0808.0691 [hep-th]].

[13] S. Kachru, R. Kallosh, A. D. Linde and S. P. Trivedi, Phys. Rev. D 68, 046005 (2003) [arXiv:hep-th/0301240].

[14] N. V. Krasnikov, Phys. Lett. B 193 (1987) 37; L. J. Dixon, "SUPERSYMMETRY BREAKING IN STRING THEORY," SLAC-PUB-5229, 1990; T. R. Taylor, Phys. Lett. B 252, 59 (1990); B. de Carlos, J. A. Casas and C. Munoz, Nucl. Phys. B 399, 623 (1993) [arXiv:hep-th/9204012]; F. Denef, M. R. Douglas and B. Florea, JHEP 0406, 034 (2004) [arXiv:hep-th/0404257].

[15] P. Svrcek and E. Witten, JHEP 0606, 051 (2006) [arXiv:hep-th/0605206]; references therein.

[16] J. P. Conlon, JHEP 0605, 078 (2006) [arXiv:hep-th/0602233].

[17] K. Choi and K. S. Jeong, JHEP 0701, 103 (2007) [arXiv:hep-th/0611279].

[18] C. G. . Callan, R. F. Dashen and D. J. Gross, Phys. Lett. B 63, 334 (1976); R. Jackiw and C. Rebbi, Phys. Rev. Lett. 37, 172 (1976); G. 't Hooft, Phys. Rev. D 14, 3432 (1976) [Erratum-ibid. D 18, 2199 (1978)].

[19] R. D. Peccei and H. R. Quinn, Phys. Rev. Lett. 38, 1440 (1977).

[20] For a review, see J. E. Kim, Phys. Rept. 150, 1 (1987); H. Y. Cheng, Phys. Rept. 158, 1 (1988); J. E. Kim and G. Carosi, Rev. Mod. Phys. 82, 557 (2010) [arXiv:0807.3125 [hep-ph]].

[21] P. Candelas, G. T. Horowitz, A. Strominger and E. Witten, Nucl. Phys. B 258, 46 (1985); P. Candelas and X. de la Ossa, Nucl. Phys. B 355, 455 (1991).

[22] R. Blumenhagen, G. Honecker and T. Weigand, JHEP 0506, 020 (2005) [arXiv:hepth/0504232]; R. Blumenhagen, G. Honecker and T. Weigand, JHEP 0508, 009 (2005) [arXiv:hep-th/0507041]; R. Blumenhagen, G. Honecker and T. Weigand, arXiv:hepth/0510050. A. Lukas, B. A. Ovrut and D. Waldram, Nucl. Phys. B 532, 43 (1998) [arXiv:hep-th/9710208]; refernces therein. G. W. Moore, G. Peradze and N. Saulina, Nucl. Phys. B 607, 117 (2001) [arXiv:hep-th/0012104]; R. Blumenhagen, S. Moster and T. Weigand, Nucl. Phys. B 751, 186 (2006) [arXiv:hep-th/0603015]; L. B. Anderson, J. Gray, A. Lukas and B. Ovrut, arXiv:1102.0011 [hep-th]; T. W. Grimm and J. Louis, 
Nucl. Phys. B 699, 387 (2004) [arXiv:hep-th/0403067]; T. W. Grimm and J. Louis, Nucl. Phys. B 718, 153 (2005) [arXiv:hep-th/0412277].

[23] M. Grana, T. W. Grimm, H. Jockers and J. Louis, Nucl. Phys. B 690, 21 (2004) [arXiv:hep-th/0312232]; H. Jockers and J. Louis, Nucl. Phys. B 705, 167 (2005) [arXiv:hepth/0409098]; H. Jockers and J. Louis, Nucl. Phys. B 718, 203 (2005) [arXiv:hepth/0502059]; T. W. Grimm, T. W. Ha, A. Klemm and D. Klevers, Nucl. Phys. B 816, 139 (2009) [arXiv:0811.2996 [hep-th]]; T. W. Grimm and D. V. Lopes, arXiv:1104.2328 [hep-th]; M. Kerstan and T. Weigand, arXiv:1104.2329 [hep-th]. F. Denef, arXiv:0803.1194 [hep-th]; A. Collinucci, F. Denef and M. Esole, JHEP 0902, 005 (2009) [arXiv:0805.1573 [hep-th]]; T. W. Grimm, Nucl. Phys. B 845, 48 (2011) [arXiv:1008.4133 [hep-th]].

[24] A. Arvanitaki, S. Dimopoulos, S. Dubovsky, N. Kaloper and J. March-Russell, Phys. Rev. D 81, 123530 (2010) [arXiv:0905.4720 [hep-th]].

[25] H. T. Janka, W. Keil, G. Raffelt and D. Seckel, Phys. Rev. Lett. 76, 2621 (1996) [arXiv:astro-ph/9507023]; W. M. Yao et al. [Particle Data Group], J. Phys. G 33, 1 (2006).

[26] B. S. Acharya, K. Bobkov and P. Kumar, JHEP 1011, 105 (2010) [arXiv:1004.5138 [hepth]].

[27] E. Komatsu et al. [WMAP Collaboration], Astrophys. J. Suppl. 192, 18 (2011) [arXiv:1001.4538 [astro-ph.CO]].

[28] T. Higaki and R. Kitano, arXiv:1104.0170 [hep-ph].

[29] For a review, see M. Grana, Phys. Rept. 423, 91 (2006) [arXiv:hep-th/0509003]; M. R. Douglas and S. Kachru, Rev. Mod. Phys. 79, 733 (2007) [arXiv:hep-th/0610102]; references therein.

[30] M. Dine, R. Rohm, N. Seiberg and E. Witten, Phys. Lett. B 156, 55 (1985); J. P. Derendinger, L. E. Ibanez and H. P. Nilles, Phys. Lett. B 155, 65 (1985); J. P. Derendinger, L. E. Ibanez and H. P. Nilles, Nucl. Phys. B 267, 365 (1986).

[31] E. Witten, Nucl. Phys. B 474, 343 (1996) [arXiv:hep-th/9604030]; For a review, see R. Blumenhagen, M. Cvetic, S. Kachru and T. Weigand, Ann. Rev. Nucl. Part. Sci. 59, 269 (2009) [arXiv:0902.3251 [hep-th]]; references therein.

[32] P. Breitenlohner and D. Z. Freedman, Annals Phys. 144, 249 (1982). 
[33] O. Lebedev, H. P. Nilles and M. Ratz, Phys. Lett. B 636, 126 (2006) [arXiv:hepth/0603047].

[34] E. Dudas, C. Papineau and S. Pokorski, JHEP 0702, 028 (2007) [arXiv:hep-th/0610297]; H. Abe, T. Higaki, T. Kobayashi and Y. Omura, Phys. Rev. D 75, 025019 (2007) [arXiv:hepth/0611024];

[35] R. Kallosh and A. D. Linde, JHEP 0702, 002 (2007) [arXiv:hep-th/0611183].

[36] H. Abe, T. Higaki and T. Kobayashi, Phys. Rev. D 76, 105003 (2007) [arXiv:0707.2671 [hep-th]].

[37] H. Abe, T. Higaki, T. Kobayashi and Y. Omura, JHEP 0804, 072 (2008) [arXiv:0801.0998 [hep-th]].

[38] L. Covi, M. Gomez-Reino, C. Gross, G. A. Palma and C. A. Scrucca, JHEP 0903, 146 (2009) [arXiv:0812.3864 [hep-th]].

[39] R. Kallosh and A. D. Linde, JHEP 0412, 004 (2004) [arXiv:hep-th/0411011].

[40] M. Gomez-Reino and C. A. Scrucca, JHEP 0605, 015 (2006) [arXiv:hep-th/0602246];

M. Gomez-Reino and C. A. Scrucca, JHEP 0609, 008 (2006) [arXiv:hep-th/0606273];

L. Covi, M. Gomez-Reino, C. Gross, J. Louis, G. A. Palma and C. A. Scrucca, JHEP 0808, 055 (2008) [arXiv:0805.3290 [hep-th]]; L. Covi, M. Gomez-Reino, C. Gross, J. Louis, G. A. Palma and C. A. Scrucca, JHEP 0806, 057 (2008) [arXiv:0804.1073 [hep-th]].

[41] R. Kitano, Phys. Lett. B 641, 203 (2006) [arXiv:hep-ph/0607090].

[42] N. Arkani-Hamed, M. Dine and S. P. Martin, Phys. Lett. B 431, 329 (1998) [arXiv:hep$\mathrm{ph} / 9803432]$.

[43] K. Choi, K. S. Jeong, K. I. Okumura and M. Yamaguchi, arXiv:1104.3274 [hep-ph].

[44] J. P. Conlon and F. G. Pedro in [9].

[45] K. Choi, H. P. Nilles, C. S. Shin and M. Trapletti in [9].

[46] R. Blumenhagen, J. P. Conlon, S. Krippendorf, S. Moster and F. Quevedo in 9].

[47] E. Dudas, Y. Mambrini, S. Pokorski, A. Romagnoni and M. Trapletti, JHEP 0903, 011 (2009) [arXiv:0809.5064 [hep-th]]. E. Dudas, Y. Mambrini, S. Pokorski and A. Romagnoni, JHEP 0804, 015 (2008) [arXiv:0711.4934 [hep-th]]. 
[48] S. Krippendorf and F. Quevedo, JHEP 0911, 039 (2009) [arXiv:0901.0683 [hep-th]].

[49] J. J. Heckman and C. Vafa, JHEP 0909, 079 (2009) [arXiv:0809.1098 [hep-th]].

[50] K. Choi and K. S. Jeong, JHEP 0608, 007 (2006) [arXiv:hep-th/0605108].

[51] R. Bousso and J. Polchinski, JHEP 0006, 006 (2000) [arXiv:hep-th/0004134].

[52] For instance, see S. Kachru, M. B. Schulz and S. Trivedi, JHEP 0310, 007 (2003) [arXiv:hepth/0201028].

[53] H. Abe, T. Higaki and T. Kobayashi, Nucl. Phys. B 742, 187 (2006) [arXiv:hep-th/0512232].

[54] H. Abe, T. Kobayashi and Y. Omura, JHEP 0711, 044 (2007) [arXiv:0708.3148 [hep-th]].

[55] A. E. Nelson and N. Seiberg, Nucl. Phys. B 416, 46 (1994) [arXiv:hep-ph/9309299].

[56] G. von Gersdorff and A. Hebecker, Phys. Lett. B 624, 270 (2005) [arXiv:hep-th/0507131]. M. Berg, M. Haack and B. Kors, JHEP 0511, 030 (2005) [arXiv:hep-th/0508043]; M. Berg, M. Haack and B. Kors, Phys. Rev. Lett. 96, 021601 (2006) [arXiv:hep-th/0508171]; K. Choi, arXiv:hep-ph/0511162; See also M. Berg, M. Haack and E. Pajer in [9]; M. Cicoli, J. P. Conlon and F. Quevedo, JHEP 0801, 052 (2008) [arXiv:0708.1873 [hep-th]] in [9];

[57] K. Bobkov, V. Braun, P. Kumar and S. Raby, JHEP 1012, 056 (2010) [arXiv:1003.1982 [hep-th]]; M. Dine, G. Festuccia, J. Kehayias and W. Wu, JHEP 1101, 012 (2011) [arXiv:1010.4803 [hep-th]].

[58] M. Haack, D. Krefl, D. Lust, A. Van Proeyen and M. Zagermann, JHEP 0701, 078 (2007) [arXiv:hep-th/0609211].

[59] R. Blumenhagen, V. Braun, T. W. Grimm and T. Weigand, Nucl. Phys. B 815, 1 (2009) [arXiv:0811.2936 [hep-th]].

[60] H. Murayama, H. Suzuki and T. Yanagida, Phys. Lett. B 291, 418 (1992); K. Choi, E. J. Chun and J. E. Kim, Phys. Lett. B 403, 209 (1997) [arXiv:hep-ph/9608222].

[61] S. Nakamura, K. i. Okumura and M. Yamaguchi, Phys. Rev. D 77, 115027 (2008) [arXiv:0803.3725 [hep-ph]].

[62] M. Endo, K. Hamaguchi and F. Takahashi, Phys. Rev. Lett. 96, 211301 (2006) [arXiv:hepph/0602061]; S. Nakamura and M. Yamaguchi, Phys. Lett. B 638, 389 (2006) [arXiv:hepph/0602081]; M. Dine, R. Kitano, A. Morisse and Y. Shirman, Phys. Rev. D 73, 123518 
(2006) [arXiv:hep-ph/0604140]; M. Kawasaki, F. Takahashi and T. T. Yanagida, Phys. Lett. B 638, 8 (2006) [arXiv:hep-ph/0603265]; T. Asaka, S. Nakamura and M. Yamaguchi, Phys. Rev. D 74, 023520 (2006) [arXiv:hep-ph/0604132]; M. Endo, K. Hamaguchi and F. Takahashi, Phys. Rev. D 74, 023531 (2006) [arXiv:hep-ph/0605091]; M. Endo, F. Takahashi and T. T. Yanagida, Phys. Rev. D 76, 083509 (2007) [arXiv:0706.0986 [hep$\mathrm{ph}]]$.

[63] M. Dine, R. Kitano, A. Morisse and Y. Shirman in 62].

[64] O. Lebedev, V. Lowen, Y. Mambrini, H. P. Nilles and M. Ratz, JHEP 0702, 063 (2007) [arXiv:hep-ph/0612035].

[65] T. Moroi and L. Randall, Nucl. Phys. B 570, 455 (2000) [arXiv:hep-ph/9906527].

[66] S. Nakamura and M. Yamaguchi, Phys. Lett. B 655, 167 (2007) [arXiv:0707.4538 [hep-ph]].

[67] T. Higaki and T. Kobayashi, Phys. Rev. D 68, 046006 (2003) [arXiv:hep-th/0304200].

[68] H. Abe, T. Higaki and T. Kobayashi, Phys. Rev. D 73, 046005 (2006) [arXiv:hepth/0511160].

[69] T. W. Grimm, M. Kerstan, E. Palti and T. Weigand, arXiv:1105.3193 [hep-th].

[70] R. Blumenhagen, S. Moster and E. Plauschinn, JHEP 0801, 058 (2008) [arXiv:0711.3389 [hep-th]].

[71] L. B. Anderson, J. Gray, A. Lukas and B. Ovrut in 22]

[72] D. S. Freed and E. Witten, arXiv:hep-th/9907189.

[73] Y. Kawamura and T. Kobayashi, Phys. Lett. B 375, 141 (1996) [Erratum-ibid. B 388, 867 (1996)] [arXiv:hep-ph/9601365]; Y. Kawamura and T. Kobayashi, Phys. Rev. D 56, 3844 (1997) [arXiv:hep-ph/9608233]; Y. Kawamura, Phys. Lett. B 446, 228 (1999) [arXiv:hepph/9811312]; T. Higaki, Y. Kawamura, T. Kobayashi and H. Nakano, Phys. Rev. D 69, 086004 (2004) [arXiv:hep-ph/0308110].

[74] C. A. Scrucca, JHEP 0712, 092 (2007) [arXiv:0710.5105 [hep-th]]. 